\title{
7. CLAY DIAGENESIS IN THE BARBADOS ACCRETIONARY COMPLEX: POTENTIAL IMPACT ON HYDROLOGY AND SUBDUCTION DYNAMICS 1,2
}

\author{
Jane Schoonmaker Tribble ${ }^{3}$
}

\begin{abstract}
The mineralogy of both bulk- and clay-sized fractions of samples from Sites 671, 672, and 674 of ODP Leg 110 was determined by X-ray diffraction. The major minerals include quartz, calcite, plagioclase feldspar, and the clay minerals smectite, illite, and kaolinite. The smectite is a dioctahedral montmorillonite and is derived primarily from degradation of volcanic ash. Percentage of smectite varies with sediment age; Miocene and Eocene sediments are the most smectiterich. High smectite content tends to correlate with elevated porosity, presumably because of the ability of smectite clays to absorb significant amounts of interlayer water. Because of a change in physical properties, the décollement zone at Site 671 formed in sediments immediately subjacent to a section of smectite-rich, high-porosity, Miocene-age sediments.

Sediments above the décollement at Site 671 , as well as all sediments analyzed from Sites 672 and 674 , contain nearly pure smectite characteristic of the alteration of volcanic ash. Within the décollement zone and underthrust sequence, however, the smectite contains up to $65 \%$ illite interlayers. Although the illite/smectite could be interpreted as detrital clay derived from South America, its absence in the sediments stratigraphically equivalent to the décollement and underthrust sequences at Sites 672 and 674 favors the interpretation that it originated by diagenetic alteration of pre-existing smectite similar to that in the overlying sediments. A significant percentage of the freshening of the pore waters observed in these zones could be due to the water released during smectite dehydration.
\end{abstract}

\section{INTRODUCTION}

In 1981, DSDP Leg 78A drilled a series of holes in the Barbados accretionary complex along a transect from Site 543 , the oceanic reference site, westward to Sites 541 and 542 (Fig. 1). These latter sites were drilled through the accreted sediments to the top of the décollement. Although the décollement was not penetrated during Leg $78 \mathrm{~A}$, sediments stratigraphically equivalent to those of the décollement were sampled at the oceanic reference site, Hole 543. A correlation was noted (Pudsey, 1984) between clay mineralogy and physical properties. Smectite-rich intervals were characterized by high porosity, high water content, and low density and shear strength. It was suggested (Pudsey, 1984; Moore and Biju-Duval, 1984) that the high-porosity, smectite-rich zones influence the location of the décollement.

Subsequent to Leg 78A drilling, a study of diagenesis associated with faulting in the accretionary complex sediments documented the presence of a pure smectite mineral along one major thrust fault and in the sediments from the very top of the décollement horizon (Schoonmaker, 1986). Interest in sampling sediments of the décollement and the underthrust series, and in determining the possible roles of high pore-fluid pressures, porosity, clay composition, and diagenesis in the dynamics of faulting and off-scraping of sediments at the toe of the accretionary complex, were among the driving forces that resulted in the return of the drilling program to the Barbados complex for a second round of drilling during ODP Leg 110.

Drilling during Leg 110 was successful in penetrating the décollement (Sites 671, 675, and 676) and the underthrust series (Site 671). An oceanic reference site (672) and two sites well up the slope of the prism (673 and 674) were also drilled (Fig. 1). One of the most intriguing results of Leg 110 was the discovery

\footnotetext{
${ }^{1}$ Moore, J. C., Mascle, A., et al., 1990. Proc. ODP, Sci. Results, 110: College Station, TX (Ocean Drilling Program).

${ }^{2}$ Hawaii Institute of Geophysics, Contribution No. 2204.

${ }^{3}$ Hawaii Institute of Geophysics, University of Hawaii, Honolulu, HI 96822.
}

of anomalous pore-fluid compositions associated with the décollement horizon and several of the thrust faults within the accreted sediments (Mascle, Moore, et al., 1988; Gieskes et al., this volume). We speculated that clay membrane filtration and/ or smectite dewatering processes might be responsible for the altered pore-fluid compositions. The pore-fluid anomalies might also reflect other diagenetic reactions occurring between the sediments and the fluids migrating through them.

The major goal of this study was to determine the clay mineralogy of the sediments, particularly those associated with thrust faults and the décollement zone. The potential influence of clay mineralogy and diagenesis on the characteristics and dynamics of the Barbados accretionary complex could then be evaluated via correlations between clay composition and physical properties, pore-fluid anomalies, and structural features.

\section{METHODS}

\section{Sample Selection}

Samples were taken on shipboard with particular emphasis on obtaining samples in close proximity to all major faults and throughout the décollement zone. In addition, samples were taken immediately adjacent to sections squeezed for pore-fluid analysis. In sampling, a preference was for clay-rich sediments. In most cases the samples analyzed for mineralogy should be representative of the depth sequences from which they came. An exception, however, are the Oligocene sediments from Sites 671 and 672 that consist of alternations of clay-rich and carbonate-rich intervals. The clay mineralogy presented here is representative of the clay-rich Oligocene intervals. Bulk mineralogy, determined on ship for all sites, is presented and discussed in the site summary chapters (Mascle, Moore, et al., 1988). Further examination of the bulk mineralogy and presentation of the clay mineralogy for Sites 671, 672, and 674 are included here.

\section{Sample Preparation}

Samples for bulk mineralogy were freeze-dried, ground to a fine powder, and packed into aluminum sample holders to yield random orientation of grains. For clay mineralogical analyses, samples were treated for removal of amorphous iron hydroxides and oxy-hydroxides using the Na-citrate, Na-dithionite treatment of Mehra and Jackson (1960). After thorough rinsing, oriented mounts were made of the bulk sample by pi- 


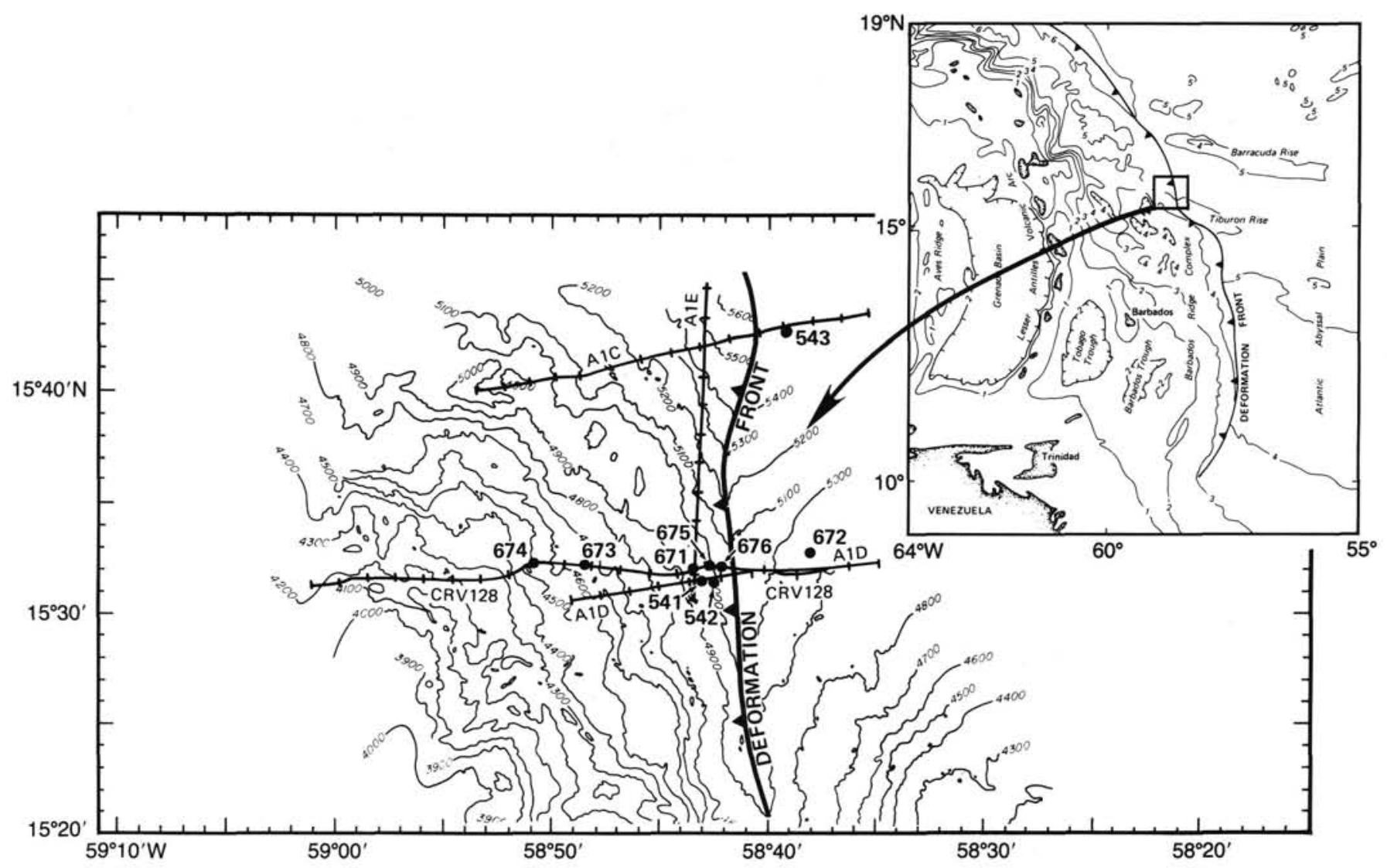

Figure 1. Location of drilling sites from DSDP Leg 78A and ODP Leg 110. The reference Sites 543 and 672 are located east of the deformation front in relatively undisturbed sediments of the Atlantic Abyssal Plain. The remaining sites form a transect through the accreted sediments westward from the deformation front. Bathymetry in meters.

petting a dilute solution of the sediment onto a glass slide. The slides were air dried, then placed in an ethylene glycol atmosphere at $40^{\circ} \mathrm{C}$ for $24 \mathrm{hr}$. The remaining sediment solution was then centifuged to isolate either the $<1$ - or $<0.5-\mu \mathrm{m}$ fraction. Oriented slides were then made following the above procedures.

\section{X-ray Diffraction Conditions}

X-ray analysis of the samples for bulk mineralogy was performed on shipboard. Analytical procedures and conditions are given in the Explanatory Notes chapter (Mascle, Moore, et al., 1988). All analyses of clay mineralogy were done on a Scintag Pad V computer-automated Xray diffraction system equipped with a solid-state Ge detector. Samples were continuously scanned from 2 to $40^{\circ} 2 \theta$ at $1^{\circ} 2 \theta / \mathrm{min}$. using $\mathrm{CuK} \alpha$ radiation. In addition, a subset of representative samples was scanned from 59 to $63^{\circ} 2 \theta$ at $0.25^{\circ} 2 \theta / \mathrm{min}$ for determination of the position of the $(060)$ peak that is diagnostic of dioctahedral vs. trioctahedral character of clays.

\section{Quantification Methods}

Percentages of quartz, plagioclase, calcite, and total clay were determined for the bulk samples following procedures outlined in the Explanatory Notes chapter (Mascle, Moore, et al., 1988). Briefly, mineralogy was quantified using Philips software APD 1700 that utilizes background-corrected, integrated peak areas. The program was calibrated using a set of six standard mineral mixtures made up to represent the approximate range of sample compositions. The total clay content determined for bulk samples was then broken down into percentages of smectite, illite, and kaolinite + chlorite using the diffraction patterns from the oriented mounts of the bulk sample. The patterns were first corrected for background using a Scintag program that subtracts the contribution of $\mathrm{K} \alpha_{2}$ radiation and then applies a fast-Fourier noise filter to the data. The resulting net intensity patterns were corrected using quartz as an internal standard. A deconvolution program that fits peaks with a Split Pearson VII function (a combination Gaussian and Lorentzian curve) was then used to calculate the integrated areas of the primary (001) peaks for smectite, illite, and the combined peak for kaolinite and chlorite. The integrated areas were multiplied by appropriate weighting factors ( 1 for smectite, 4 for illite, and 2 for kaolinite + chlorite; Mann and Müller, 1979) and normalized to the total clay content for the individual sample. Similar procedures were used to quantify the clay-sized fraction, but the percentages of smectite, illite, and kaolinite + chlorite were normalized to 100 . Accuracy of relative percentages of minerals determined by X-ray diffraction quantification is estimated to be $\pm 10 \%$. In addition to these major phases, the presence of accessory minerals was noted as either trace or significant (compared to the major minerals) in quantity.

\section{Composition of the Smectite}

Clay minerals of the smectite group commonly exhibit varying degrees of mixed layering. That is, individual sheets within the phyllosilicate structure have a different chemical composition and structure from that of the smectite. The best studied mixed-layer mineral is illite/smectite, which consists of illite and smectite layers interstratified in proportions ranging from $100 \%$ smectite to $100 \%$ illite. An increase in the percentage of interstratified illite during diagenesis has been documented at numerous locations and is commonly associated with burial (e.g., Perry and Hower, 1970; Weaver and Beck, 1971; Hower et al., 1976; Boles and Franks, 1979) or more rarely with contact metamorphism or hydrothermal alteration (Nadeau and Reynolds, 1981; Horton, 1983; Vergo, 1984). Most smectite in the Leg 110 sediments contains a low percentage of interstratified illite and is referred to in this work simply as smectite. The term illite/smectite is reserved for diagenetically altered smectites containing significant amounts of interstratified illite (see discussion below). The percentages of illite reported in Tables 1, 2, and 3 and Figure 3 refer only to discrete illite; illite interstratified in smectite is not included in these percentages. 
The development of mixed layering in smectite causes a change in lattice dimensions that is reflected in a shift in peak position on an Xray diffraction pattern. This shift has been quantified for the illite/ smectites (Reynolds and Hower, 1970). A smooth curve was fit to the peak position vs. composition data of Reynolds and Hower, and the degree of mixed layering in the smectites from Leg 110 was determined from the position of the illite/smectite $(001 / 002)$ peak. The $(002 / 003)$ peak was broader and less well defined, so it was not used. These estimates of composition are probably accurate to within $\pm 5 \%$ interlayers. In a few samples the presence of accessory minerals that have peaks near the (001/002) peak for smectite (e.g., amphibole and clinoptilolite) made precise location of the smectite peak difficult; for those samples, accuracy is probably only $\pm 10 \%$ interlayers.

\section{MINERALOGY: RESULTS AND DISCUSSION}

\section{Bulk Mineralogy}

The bulk mineralogies of samples from Holes 671B, 672A, and $674 \mathrm{~A}$ are presented in Tables 1,2 , and 3 . The calcite content of the sediments varies significantly with depth at all three sites. In general, Pliocene-Pleistocene sediments are fairly calcareous, whereas Miocene and upper Oligocene sediments contain little if any calcite. The lower Oligocene and Eocene sequences of Holes $671 \mathrm{~B}$ and $672 \mathrm{~A}$ consist of alternations of calcite-rich and calcite-free sediments, whereas at Hole 674A the middle Eocene is represented by calcareous sediments and the lower Oligocene is calcite-free. The changes in percentage of total clay of these sediments largely reflect the changes in calcite content. Calcitefree sediments generally contain $70-90 \%$ total clay.

Plagioclase content tends to decrease with depth at all three sites. With the exception of a few very plagioclase-rich samples, the percentage of plagioclase also appears to decrease slightly from Sites 674 to 671 to 672 . The source of the plagioclase is most likely volcanic ash from the Lesser Antilles Arc. The decrease in plagioclase content with depth reflects a general increase in age with depth (despite repetition of section via faulting) and, thus, both an increase in distance of the site of deposition from the arc, and an increase in diagenetic alteration of the ash with age. The slight decrease in plagioclase content from Hole $674 \mathrm{~A}$ eastward to Hole $672 \mathrm{~A}$ for any given age probably reflects the originally greater east-west separation of the sites and their different distances from the volcanic arc.

Quartz content of the sediments at these three sites shows no net trend with depth. Although the percentage of quartz does show significant variations, most samples contain less than $20 \%$ quartz.

\section{Variations in Clay Mineralogy with Age}

The percentages of the major clay minerals are given in Tables 1, 2, and 3. Although kaolinite and chlorite were not differentiated quantitatively, this fraction is composed predominantly of kaolinite. Calculations for the samples with the largest chlorite peaks indicate that chlorite makes up less than $5 \%$ of the total clay content. Representative diffraction profiles for several samples are shown in Figure 2. Variations in the distributions of the clay minerals between sites and with age are illustrated in the triangular plots of Figure 3. The clay mineral distributions are similar at all sites. The Pliocene-Pleistocene sediments of Hole $674 \mathrm{~A}$ appear to be more smectitic than their counterparts at Holes $671 \mathrm{~B}$ and $672 \mathrm{~A}$, although interpretation of Hole $674 \mathrm{~A}$ is limited by the number of samples analyzed. Several trends in the distribution with age are evident from these plots. Sediments of Pliocene-Pleistocene age have relatively high kaolinite + chlorite and illite contents. Miocene sediments are characterized by a wide range of compositions, but in general are more smectiterich. The Oligocene sediments also display a wide range of compositions, but tend to have lower proportions of illite than the younger sediments. Eocene-age sediments, represented only at Sites 672 and 674, have distinctly low illite contents.
These variations in clay mineral distribution with age reflect the balance between delivery of terrigenous-derived material and deposition of volcanic ash at each site. Kaolinite, chlorite, and illite are derived predominantly from South America (Wright, 1984; Pudsey, 1984; Schoonmaker et al., 1986). Smectite in marine sediments may be derived from either continental weathering (South America) or from submarine alteration of volcanic ash (from the Lesser Antilles Arc). As we discuss below, composition of the smectite can help differentiate between these two sources, and it appears likely that most of the smectite in the Leg 110 sediments originated from degradation of ash. Both of these sediment sources fluctuate in magnitude with time. Natland (1984) documented the discontinuous nature of ash deposition for the Leg 78A sediments. In addition, the diagenetic alteration of ash to smectite requires time, so an increase in smectite content of older sediments is not unexpected. The delivery of terrigenous sediments from South America also fluctuates depending on such factors as sea-level variations, rates of weathering which are largely climate-controlled, and ocean-current activity. Although most of the variations in clay mineral distributions are probably explained by the balance between the two major sediment sources, there does appear to be a decrease with age in the proportion of illite relative to kaolinite + chlorite delivered from South America.

\section{Correlation of Smectite Content with Other Sediment Characteristics}

Depth distributions of smectite for Holes 671B and 672A are plotted in Figures 4 and 5 along with the variation in total clay content, porosity, and location of major faults. Although porosity measurements were not always made on the same samples used for mineralogical analyses, the porosity values of Figures 4 and 5 are for samples located as close as possible to the mineralogical samples, in most cases within a meter. In Hole 671B, samples from the depth range $400-500$ mbsf have relatively high smectite contents (Fig. 4). These sediments are Miocene in age as noted in Figure 3. Most of the major thrust faults and the décollement are located in sediments of relatively low smectite content, directly underlying local maxima in percentage of smectite. This trend is particularly notable for the décollement that is located in sediments immediately below the Miocene high-smectite section. The decrease in smectite content at the depth of the décollement does not simply represent a decrease in total clay content. Although total clay does decrease slightly in this depth range (Fig. 4), the percentages of the other clay minerals increase at the expense of smectite (Table 1).

Comparison of the depth trend of percent smectite with that of porosity in Hole 671B shows that smectite-rich sediments generally have relatively high porosities, particularly those overlying the décollement zone. This correlation of smectite content and porosity probably reflects the high water content of smectite minerals, which adsorb water on grain surfaces and edges as well as in interlayer positions. Similar correlations between porosity and décollement location were noted for DSDP Leg 78A (Marlow et al., 1984) and for Leg 110 (Wilkens et al., this volume) on the basis of many more porosity analyses.

Examination of the relationship between smectite and total clay content, porosity, and faulting in Hole 672A (Fig. 5) shows no significant correlation between a zone of normal faulting at about 100 mbsf and deviations in the depth trends of these sediment properties. The section that is stratigraphically equivalent to the décollement at Site 671 (the proto-décollement), however, lies just beneath a zone of high smectite content as was the case in Hole $671 \mathrm{~B}$. In contrast to Hole $671 \mathrm{~B}$, however, the total clay content of sediments within the proto-décollement is significantly lower than that of the overlying section. The decrease in clay content reflects primarily the lower smectite content because the percentages of other clay minerals change only slightly 
Table 1. Bulk and clay mineralogy, Leg 110, Hole 671B.

\begin{tabular}{|c|c|c|c|c|c|c|c|c|c|c|c|c|c|c|c|c|c|c|c|c|}
\hline \multirow[t]{2}{*}{ SAMPLE ${ }^{a}$} & \multirow{2}{*}{$\begin{array}{l}\text { DEPTH } \\
\text { (mbsf) }\end{array}$} & \multirow[t]{2}{*}{ AGE } & \multicolumn{4}{|c|}{ BULK MINERALOGYb } & \multicolumn{3}{|c|}{ BULK CLAY MINERALOGYC } & \multicolumn{3}{|c|}{ CLAY-SIZED FRACTION $^{d}$} & \multicolumn{8}{|c|}{ ACCESSORY MINERALS ${ }^{\mathrm{e}}$} \\
\hline & & & QTZ & PLAG & CAL & CLAY & SM & IL & $\mathrm{K}+\mathrm{C}$ & SM & IL & $\mathrm{K}+\mathrm{C}$ & PALY & CLIN & GIBB & OP-CT & $\mathrm{DOL}$ & RHOD & AMPH & PYX \\
\hline $1-3 / 140-144$ & 4 & E.PLEIS & 13 & 7 & 27 & 53 & 16 & 16 & 21 & 44 & 25 & 32 & & & & & & & $\mathrm{x}$ & \\
\hline $2-5 / 140-144$ & 15 & E.PLEIS & 14 & 23 & 5 & 57 & 18 & 15 & 25 & 32 & 32 & 36 & & & & & & & $\mathrm{x}$ & \\
\hline $3-3 / 129-134$ & 21 & E.PLEIS & 11 & 9 & 51 & 29 & 7 & 9 & 13 & 27 & 33 & 39 & & & & & $\mathrm{x}$ & & $\mathrm{x}$ & \\
\hline $4-5 / 140-144$ & 34 & L.PLIOC & 1 & 31 & 0 & 67 & 14 & 22 & 31 & 30 & 33 & 37 & & & $\mathrm{x}$ & & & & $\mathrm{X}$ & \\
\hline $6-4 / 140-145$ & 51 & L.PLIOC & 18 & 12 & 41 & 29 & 10 & 5 & 13 & 28 & 22 & 50 & & & & & & $\mathrm{x}$ & $\mathrm{X}$ & \\
\hline $7-3 / 126-128$ & 59 & L.PLIOC & 10 & 1 & 59 & 30 & 9 & 6 & 15 & 52 & 19 & 29 & & & & & & $\mathrm{x}$ & $\mathrm{x}$ & \\
\hline $8-4 / 138-143$ & 70 & L.PLIOC & 7 & 37 & 18 & 38 & 7 & 14 & 17 & 35 & 30 & 35 & & & $\mathrm{x}$ & & & & $\mathrm{x}$ & \\
\hline $9-2 / 131-134$ & 77 & E.PLIOC & 5 & 2 & 0 & 93 & 36 & 19 & 38 & 49 & 15 & 36 & & & & & & & & \\
\hline $10-3 / 125-130$ & 88 & E.PLIOC & 9 & 0 & 24 & 67 & 23 & 18 & 26 & 51 & 18 & 32 & & & & & & & & \\
\hline $11-4 / 118-120$ & 97 & E.PLIOC & 10 & 6 & 34 & 50 & 15 & 13 & 21 & 58 & 16 & 25 & & & & & & $\mathrm{x}$ & & \\
\hline $12-5 / 121-123$ & 108 & E.PLIOC & 10 & 8 & 23 & 59 & 20 & 15 & 25 & 40 & 24 & 35 & & & $\mathrm{x}$ & & & & $\mathrm{x}$ & \\
\hline $13-3 / 141-145$ & 115 & L.MIOC & 3 & 6 & 0 & 91 & 39 & 22 & 30 & 36 & 30 & 34 & & & & & & & & $\mathrm{x}$ \\
\hline $14-6 / 86-91$ & 128 & E.PLEIS & 0 & 18 & 0 & 81 & 24 & 28 & 30 & 42 & 26 & 33 & & & $\mathrm{x}$ & & & $\mathrm{xx}$ & & \\
\hline $14-6 / 111-116$ & 129 & E.PLEIS & 11 & 29 & 11 & 49 & 20 & 14 & 15 & 45 & 31 & 24 & & & $\mathrm{x}$ & & & & & \\
\hline $15-1 / 48-50$ & 130 & E.PLEIS & 11 & 29 & 0 & 60 & 23 & 19 & 18 & 37 & 33 & 30 & & & $\mathrm{x}$ & & & & $\mathrm{x}$ & \\
\hline $16-7 / 22-27$ & 148 & E.PLEIS & 11 & 10 & 20 & 60 & 12 & 19 & 29 & 40 & 29 & 31 & & & & & & $\mathrm{x}$ & $\mathrm{X}$ & \\
\hline $17-4 / 138-143$ & 154 & E.PLEIS & 11 & 23 & 9 & 58 & 13 & 23 & 22 & 45 & 32 & 23 & & & $\mathrm{x}$ & & & & $\mathrm{x}$ & \\
\hline $18-4 / 30-35$ & 163 & E.PLEIS & 14 & 10 & 43 & 33 & 4 & 14 & 15 & 38 & 32 & 30 & & & & & & & $\mathrm{x}$ & $x$ \\
\hline $19-1 / 139-144$ & 169 & E.PLEIS & 14 & 11 & 69 & 6 & 1 & 2 & 2 & 39 & 31 & 31 & & & & & & & $\mathrm{x}$ & \\
\hline $21-2 / 90-94$ & 189 & E.PLEIS & 11 & 5 & 28 & 56 & 15 & 19 & 22 & 38 & 36 & 26 & & & & & & & $\mathrm{x}$ & \\
\hline $22-5 / 139-143$ & 203 & E.PLEIS & 11 & 5 & 40 & 44 & 8 & 11 & 24 & 49 & 17 & 35 & & & $\mathrm{x}$ & & & $\mathrm{xx}$ & $\mathrm{x}$ & \\
\hline $25-4 / 136-141$ & 230 & L.PLIOC & 0 & 20 & 0 & 80 & 13 & 20 & 48 & 54 & 10 & 36 & & & & & & $\mathrm{xx}$ & $\mathrm{x}$ & $\mathrm{x}$ \\
\hline $27-2 / 60-65$ & 246 & L.PLIOC & 0 & 5 & 0 & 96 & 33 & 27 & 36 & 48 & 23 & 29 & & & & & & & $\mathrm{x}$ & \\
\hline $28-5 / 137-142$ & 260 & L.PLIOC & 11 & 9 & 46 & 35 & 7 & 12 & 16 & 52 & 22 & 26 & & & & & & & & \\
\hline $30-3 / 121-123$ & 276 & E.PLIOC & 9 & 3 & 7 & 82 & 18 & 23 & 40 & 28 & 31 & 42 & & & $\mathrm{x}$ & & & $\mathrm{x}$ & $\mathrm{x}$ & \\
\hline $32-5 / 85-90$ & 298 & E.PLIOC & 3 & 77 & 2 & 17 & 11 & 2 & 4 & 81 & 5 & 14 & & $\mathrm{x}$ & & & & & & \\
\hline $33-2 / 76-81$ & 303 & E.PLIOC & 9 & 4 & 12 & 76 & 16 & 25 & 35 & 32 & 36 & 32 & & & & & & & & \\
\hline $34-3 / 135-140$ & 310 & E.PLIOC & 0 & 11 & 0 & 89 & 78 & 4 & 7 & 88 & 3 & 8 & & & & & & & $\mathrm{X}$ & \\
\hline $37-2 / 131-133$ & 337 & L.MIOC & 11 & 2 & 19 & 68 & 26 & 16 & 26 & 41 & 28 & 31 & & & $\mathrm{x}$ & & & & & $\mathrm{x}$ \\
\hline $39-\mathrm{CC} / 17-19$ & 354 & L.MIOC & 21 & 17 & 8 & 54 & 27 & 9 & 18 & 55 & 16 & 29 & & $\mathrm{x}$ & $\mathrm{x}$ & & & & $\mathrm{x}$ & \\
\hline $40-3 / 136-140$ & 367 & L.MIOC & 11 & 8 & 6 & 75 & 21 & 22 & 31 & 57 & 17 & 26 & & & & & & & $\mathbf{X}$ & \\
\hline $41-4 / 62-67$ & 378 & L.MIOC & 30 & 13 & 13 & 44 & 18 & 9 & 16 & 61 & 15 & 25 & $\mathrm{x}$ & & & & & $\mathrm{x}$ & $\mathrm{x}$ & $\mathrm{x}$ \\
\hline $41-4 / 101-103$ & 378 & L.MIOC & 21 & 5 & 9 & 65 & 15 & 22 & 28 & 47 & 18 & 35 & & & & & & & $\mathrm{x}$ & \\
\hline $41-5 / 15-20$ & 379 & L.MIOC & 13 & 6 & 34 & 47 & 5 & 23 & 19 & 40 & 27 & 34 & & & $\mathrm{x}$ & & & & & \\
\hline $42-4 / 33-40$ & 383 & L.MIOC & 13 & 3 & 19 & 65 & 15 & 22 & 28 & 38 & 30 & 32 & & & $\mathrm{x}$ & & & $\mathrm{xX}$ & $\mathrm{x}$ & \\
\hline $42-6 / 83-88$ & 386 & L.MIOC & 8 & 19 & 16 & 57 & 19 & 16 & 22 & 66 & 10 & 25 & & & $\mathrm{x}$ & & & $\mathrm{x}$ & $\mathrm{x}$ & \\
\hline $43-2 / 40-45$ & 389 & $?$ & 9 & 17 & 0 & 75 & 55 & 5 & 15 & 77 & 6 & 17 & & $\mathrm{X}$ & & & & & & \\
\hline
\end{tabular}




\begin{tabular}{|c|c|c|c|c|c|c|c|c|c|c|c|c|c|c|c|c|c|c|c|c|}
\hline $43-3 / 135-140$ & 392 & $?$ & 28 & 14 & 0 & 58 & 39 & 5 & 14 & 61 & 15 & 24 & & & & & & $\mathrm{x}$ & $\mathrm{x}$ & \\
\hline $44-4 / 104-107$ & 402 & $?$ & 13 & 20 & 0 & 67 & 52 & 4 & 11 & 79 & 6 & 15 & & $\mathrm{x}$ & $\mathrm{x}$ & & & $\mathrm{x}$ & $\mathrm{x}$ & \\
\hline $45-3 / 62-67$ & 410 & $?$ & 7 & 36 & 0 & 57 & 45 & 4 & 8 & 84 & 3 & 12 & & & & & & & $\mathrm{x}$ & \\
\hline $46-3 / 135-140$ & 420 & L.MIOC & 8 & 10 & 1 & 81 & 68 & 2 & 12 & 78 & 6 & 16 & & & & & & & & \\
\hline $48-4 / 134-136$ & 441 & $?$ & 10 & 9 & 1 & 79 & 54 & 4 & 21 & 75 & 4 & 21 & & & & & & & $\mathrm{x}$ & \\
\hline $49-2 / 137-140$ & 447 & $?$ & 9 & 4 & 0 & 87 & 42 & 10 & 34 & 60 & 6 & 34 & & & & & & & $\mathrm{x}$ & \\
\hline $50-1 / 123-126$ & 455 & ? & 10 & 4 & 0 & 86 & 40 & 9 & 36 & 50 & 14 & 36 & & & $\mathrm{x}$ & & & $\mathrm{x}$ & $\mathrm{x}$ & \\
\hline $51-2 / 110-115$ & 466 & ? & 8 & 4 & 0 & 88 & 75 & 3 & 10 & 90 & 0 & 10 & & & & & & & $\mathrm{x}$ & \\
\hline $52-2 / 134-140$ & 476 & ? & 4 & 5 & 0 & 91 & 66 & 4 & 20 & 81 & 4 & 15 & & & & & & & $\mathrm{x}$ & \\
\hline $52-4 / 62-67$ & 478 & ? & 0 & 6 & 0 & 94 & 76 & 2 & 16 & 93 & 0 & 7 & & & & & & & & \\
\hline $54-3 / 104-106$ & 496 & $?$ & 0 & 16 & 0 & 84 & 63 & 5 & 16 & 83 & 2 & 14 & $\mathrm{x}$ & & & & & & $\mathrm{x}$ & \\
\hline $54-4 / 104-106$ & 497 & ? & 2 & 12 & 0 & 86 & 74 & 2 & 9 & 90 & 2 & 8 & & & & & & & $\mathrm{x}$ & \\
\hline $55-4 / 72-74$ & 506 & $?$ & 0 & 14 & 0 & 86 & 61 & 7 & 18 & 79 & 3 & 18 & & & & & & & & \\
\hline $55-5 / 134-137$ & 508 & ? & 12 & 11 & 0 & 77 & 51 & 4 & 22 & 64 & 7 & 29 & & & $\mathrm{x}$ & & & & $\mathrm{x}$ & \\
\hline $56-2 / 134-137$ & 514 & ? & 17 & 9 & 0 & 74 & 27 & 5 & 42 & 51 & 5 & 44 & & & $\mathrm{x}$ & & & & $\mathrm{x}$ & \\
\hline $56-3 / 56-59$ & 514 & ? & 11 & 4 & 0 & 85 & 40 & 5 & 41 & 57 & 4 & 39 & & & $\mathrm{x}$ & & & $\mathrm{x}$ & $\mathrm{x}$ & \\
\hline $56-4 / 75-78$ & 516 & $?$ & 18 & 4 & 0 & 78 & 34 & 6 & 38 & 55 & 6 & 40 & & & & & & $\mathrm{x}$ & $\mathrm{x}$ & \\
\hline $58-5 / 135-140$ & 537 & L.OLIGO & 26 & 3 & 0 & 72 & 25 & 15 & 32 & 34 & 26 & 41 & & & & & & & & \\
\hline $60-2 / 89-94$ & 551 & L.OLIGO & 16 & 0 & 0 & 84 & 42 & 2 & 40 & 57 & 1 & 41 & & & & & & & $\mathrm{x}$ & $\mathrm{x}$ \\
\hline $61-5 / 135-140$ & 566 & E.OLIGO & 27 & 0 & 0 & 73 & 21 & 16 & 36 & 31 & 18 & 51 & & & & $\mathrm{x}$ & & $\mathrm{x}$ & & \\
\hline $63-5 / 135-139$ & 584 & E.OLIGO & 25 & 2 & 0 & 72 & 21 & 19 & 32 & 28 & 33 & 39 & & & & $\mathrm{x}$ & & & $\mathrm{x}$ & \\
\hline $64-6 / 56-60$ & 595 & E.OLIGO & 19 & 0 & 0 & 81 & 53 & 11 & 17 & 69 & 16 & 15 & & & & & & & & $\mathrm{x}$ \\
\hline $65-5 / 134-138$ & 604 & E.OLIGO & 18 & 0 & 0 & 82 & 30 & 19 & 33 & 41 & 23 & 35 & & & & & & $\mathrm{x}$ & $\mathrm{x}$ & \\
\hline $66-2 / 29-34$ & 607 & E.OLIGO & 11 & 0 & 0 & 89 & 54 & 14 & 21 & 61 & 21 & 17 & & & & & & $\mathrm{x}$ & $\mathrm{x}$ & \\
\hline $66-4 / 85-90$ & 611 & E.OLIGO & 26 & 1 & 8 & 66 & 23 & 7 & 36 & 42 & 12 & 46 & & & & & & & & \\
\hline $67-2 / 26-31$ & 617 & E.OLIGO & 5 & 0 & 42 & 53 & 19 & 2 & 32 & 32 & 5 & 63 & & & & & & $\mathrm{x}$ & $\mathrm{x}$ & \\
\hline $67-5 / 127-130$ & 622 & E.OLIGO & 17 & 0 & 0 & 82 & 18 & 21 & 44 & 23 & 27 & 51 & & & & & & $\mathrm{x}$ & & \\
\hline $70-2 / 40-45$ & 646 & E.OLIGO & 10 & 1 & 0 & 89 & 11 & 39 & 39 & 10 & 46 & 44 & & & $\mathrm{x}$ & $\mathrm{x}$ & & & $\mathrm{x}$ & \\
\hline $70-4 / 135-140$ & 650 & E.OLIGO & 12 & 0 & 0 & 88 & 25 & 23 & 41 & 31 & 29 & 40 & & & & & & $\mathrm{x}$ & & \\
\hline $71-3 / 47-50$ & 657 & E.OLIGO & 14 & 0 & 8 & 78 & 43 & 12 & 23 & 57 & 18 & 26 & & & & & & $\mathrm{x}$ & & \\
\hline $71-5 / 80-83$ & 660 & E.OLIGO & 14 & 0 & 0 & 86 & 56 & 6 & 24 & 67 & 8 & 25 & & & & & $\mathrm{x}$ & $\mathrm{x}$ & & \\
\hline $72-5 / 35-38$ & 669 & E.OLIGO & 10 & 2 & 0 & 88 & 70 & 6 & 12 & 80 & 9 & 11 & & & & & & & $\mathrm{x}$ & \\
\hline $73-5 / 137-140$ & 680 & $?$ & 11 & 3 & 0 & 86 & 51 & 3 & 33 & 57 & 5 & 38 & $\mathrm{x}$ & & & & & $\mathrm{x}$ & $\mathrm{x}$ & \\
\hline
\end{tabular}

${ }^{2}$ Sample identification: core-section/cm interval.

b Bulk mineralogy reported as percents of quartz (QTZ), plagioclase (PLAG), calcite (CAL), and total clay minerals (CLAY), normalized to 100.

${ }^{c}$ Bulk clay mineralogy reported as percents of smectite (SM), illite (IL), and kaolinite plus chlorite $(\mathrm{K}+\mathrm{C})$, normalized to total clay content.

$\mathrm{dClay}$ fraction mineralogy reported as percents smectite (SM), illite (IL), and kaolinite plus chlorite (K+C), normalized to 100.

e Presence of accessory minerals clinoptilolite (CLIN), gibbsite (GIBB), rhodochrosite (RHOD), amphibole (AMPH), and pyroxene (PYX) indicated as trace (X) or minor (XX)

as compared to major constituents. 
Table 2. Bulk and clay mineralogy, Leg 110, Hole 672A.

\begin{tabular}{|c|c|c|c|c|c|c|c|c|c|c|c|c|c|c|c|c|c|c|c|c|}
\hline \multirow[t]{2}{*}{ SAMPLE } & \multirow{2}{*}{$\begin{array}{l}\text { DEPTH } \\
\text { (mbsf) }\end{array}$} & \multirow[t]{2}{*}{ AGE } & \multicolumn{4}{|c|}{ BULK MINERALOGY } & \multicolumn{3}{|c|}{ BULK CLAY MINERALOGY } & \multicolumn{3}{|c|}{ CLAY-SIZED FRACTION } & \multicolumn{8}{|c|}{ ACCESSORY MINERALS } \\
\hline & & & QTZ & PLAG & $\mathrm{CAL}$ & CLAY & SM & IL & $\mathrm{K}+\mathrm{C}$ & SM & IL & $\mathrm{K}+\mathrm{C}$ & PALY & CLIN & GIBB & OP-CT & $\mathrm{DOL}$ & RHOD & AMPH & PYX \\
\hline $2-6 / 140-145$ & 12 & E.PLEIS & 9 & 50 & 2 & 39 & 15 & 8 & 16 & 37 & 31 & 32 & & & & & & & $\mathrm{x}$ & \\
\hline $3-4 / 140-145$ & 19 & E.PLEIS & 18 & 6 & 51 & 25 & 8 & 7 & 10 & 36 & 32 & 32 & & & $\mathrm{x}$ & & & $\mathrm{x}$ & $\mathrm{x}$ & \\
\hline $4-5 / 140-145$ & 30 & E.PLEIS & 13 & 12 & 12 & 63 & 13 & 23 & 26 & 38 & 28 & 35 & & & $\mathrm{x}$ & & & $\mathrm{x}$ & $\mathrm{x}$ & \\
\hline $5-5 / 136-140$ & 39 & L.PLIOC & 12 & 12 & 48 & 28 & 7 & 7 & 15 & 42 & 26 & 33 & & & $\mathrm{x}$ & & & $\mathrm{x}$ & $\mathrm{x}$ & \\
\hline $7-5 / 140-144$ & 58 & L.PLIOC & 11 & 0 & 50 & 39 & 17 & 9 & 13 & 60 & 15 & 25 & & & & & & $\mathrm{x}$ & $\mathrm{x}$ & \\
\hline $9-5 / 141-145$ & 77 & L.PLIOC & 9 & 5 & 42 & 43 & 12 & 11 & 20 & 47 & 17 & 35 & & & & & & $\mathrm{x}$ & $\mathrm{x}$ & \\
\hline $12-5 / 139-144$ & 106 & E.PLIOC & 14 & 5 & 17 & 64 & 15 & 23 & 26 & 44 & 23 & 33 & & & $\mathrm{x}$ & & & $x x$ & & \\
\hline $13-3 / 135-140$ & 112 & L.MIOC & 9 & 7 & 3 & 81 & 35 & 20 & 26 & 49 & 19 & 32 & & $\mathrm{x}$ & $\mathrm{x}$ & & & & $\mathrm{x}$ & \\
\hline $14-3 / 76-82$ & 121 & L.MIOC & 11 & 3 & 11 & 74 & 29 & 23 & 23 & 48 & 19 & 33 & & $\mathrm{x}$ & & & & $\mathrm{x}$ & $\mathrm{x}$ & \\
\hline $15-3 / 134-139$ & 128 & L.MIOC & 49 & 2 & 0 & 49 & 23 & 9 & 17 & 53 & 20 & 27 & & $\mathrm{x}$ & $\mathrm{x}$ & & & $\mathrm{x}$ & $\mathrm{x}$ & $\mathrm{x}$ \\
\hline $17-4 / 115-120$ & 148 & L.MIOC & 6 & 7 & 0 & 87 & 57 & 7 & 23 & 68 & 6 & 26 & $\mathrm{x}$ & & $\mathrm{x}$ & & & $\mathrm{x}$ & $\mathrm{x}$ & \\
\hline $18-3 / 135-140$ & 156 & L.MIOC & 3 & 7 & 0 & 90 & 60 & 4 & 26 & 69 & 4 & 27 & & & & & & $\mathrm{x}$ & $\mathrm{x}$ & \\
\hline $19-3 / 135-139$ & 166 & $?$ & 5 & 4 & 0 & 91 & 63 & 5 & 23 & 76 & 2 & 21 & & & & & & & & \\
\hline $20-2 / 109-114$ & 173 & $?$ & 9 & 27 & 0 & 63 & 55 & 2 & 6 & 91 & 2 & 8 & & $\mathrm{x}$ & & & & $\mathrm{x}$ & $\mathrm{x}$ & \\
\hline $21-2 / 134-138$ & 183 & E.MIOC & 23 & 0 & 0 & 76 & 33 & 10 & 34 & 51 & 10 & 39 & $\mathrm{x}$ & & & & & $\mathrm{x}$ & $\mathrm{x}$ & \\
\hline $21-3 / 72-76$ & 184 & E.MIOC & 11 & 25 & 0 & 64 & 38 & 7 & 20 & 47 & 12 & 40 & & $\mathrm{x}$ & & & & $\mathrm{x}$ & & \\
\hline $22-2 / 107-110$ & 192 & E.MIOC & 5 & 8 & 0 & 86 & 43 & 8 & 35 & 66 & 6 & 28 & & & & & & $\mathrm{x}$ & $\mathrm{x}$ & \\
\hline $22-5 / 104-106$ & 197 & E.MIOC & 2 & 5 & 0 & 92 & 65 & 4 & 23 & 78 & 2 & 20 & & $\mathrm{x}$ & & & & $\mathrm{x}$ & $\mathrm{x}$ & \\
\hline $23-1 / 36-40$ & 200 & E.MIOC & 10 & 1 & 0 & 88 & 33 & 12 & 43 & 46 & 9 & 45 & & & & & & & & \\
\hline $23-6 / 120-125$ & 208 & E.MIOC & 2 & 2 & 0 & 95 & 44 & 8 & 43 & 59 & 5 & 35 & & & & & & $\mathrm{x}$ & $\mathrm{x}$ & \\
\hline $24-5 / 135-140$ & 216 & L.OLIGO & 11 & 3 & 0 & 85 & 35 & 15 & 35 & 54 & 9 & 36 & & & & & & $\mathrm{x}$ & $\mathrm{x}$ & \\
\hline $27-5 / 135-140$ & 245 & L.OLIGO & 12 & 0 & 0 & 88 & 29 & 12 & 46 & 37 & 9 & 54 & & $\mathrm{x}$ & & & & $\mathrm{x}$ & $\mathrm{x}$ & \\
\hline $29-4 / 130-135$ & 262 & E.OLIGO & 9 & 1 & 23 & 68 & 43 & 2 & 23 & 62 & 2 & 37 & & $\mathrm{x}$ & & & & & $\mathrm{x}$ & \\
\hline $30-5 / 136-139$ & 273 & E.OLIGO & 20 & 2 & 0 & 78 & 25 & 21 & 32 & 36 & 24 & 40 & & & $\mathrm{x}$ & & & $\mathrm{x}$ & $\mathrm{x}$ & \\
\hline $31-3 / 82-85$ & 279 & E.OLIGO & 10 & 0 & 42 & 48 & 17 & 4 & 28 & 43 & 4 & 53 & & & & & & $\mathrm{x}$ & $\mathrm{x}$ & \\
\hline $32-4 / 137-139$ & 291 & E.OLIGO & 39 & 4 & 0 & 56 & 24 & 8 & 24 & 50 & 11 & 39 & & & & & & $\mathrm{x}$ & $\mathrm{x}$ & \\
\hline $34-4 / 134-139$ & 310 & E.OLIGO & 3 & 0 & 66 & 32 & 14 & 3 & 14 & 47 & 3 & 50 & & & & & & $\mathrm{x}$ & & \\
\hline $38-5 / 123-128$ & 349 & $?$ & 7 & 4 & 0 & 89 & 51 & 7 & 31 & 58 & 5 & 37 & & & & & & $\mathrm{x}$ & $\mathrm{x}$ & \\
\hline $39-2 / 142-145$ & 354 & L.EOCENE & 56 & 0 & 1 & 44 & 27 & 1 & 16 & 67 & 1 & 32 & & $\mathrm{x}$ & & & & $\mathrm{x}$ & & \\
\hline $40-5 / 140-143$ & 368 & L.EOCENE & 4 & 2 & 0 & 93 & 60 & 2 & 31 & 61 & 2 & 36 & & & & & & $\mathrm{X}$ & $\mathrm{X}$ & \\
\hline $41-1 / 141-144$ & 372 & M.EOCENE & 10 & 3 & 1 & 87 & 53 & 2 & 31 & 67 & 3 & 31 & & & & & & $\mathrm{x}$ & $\mathrm{x}$ & \\
\hline $42-3 / 0-3$ & 383 & M.EOCENE & 20 & 0 & 19 & 61 & 29 & 1 & 31 & 59 & 3 & 37 & & & & & & $\mathrm{x}$ & $\mathrm{x}$ & \\
\hline $43-4 / 137-142$ & 395 & M.EOCENE & 9 & 5 & 1 & 86 & 53 & 3 & 30 & 58 & 3 & 39 & & $\mathrm{x}$ & & $\mathrm{x}$ & & & $\mathrm{x}$ & \\
\hline $46-1 / 113-118$ & 419 & M.EOCENE & 34 & 0 & 27 & 39 & 30 & 0 & 9 & 65 & 1 & 33 & & & & & & $\mathbf{X}$ & $\mathrm{x}$ & \\
\hline $48-3 / 102-107$ & 441 & M.EOCENE & 7 & 1 & 10 & 82 & 37 & 3 & 42 & 44 & 3 & 53 & & & & & & & $\mathrm{x}$ & \\
\hline $51-2 / 136-140$ & 468 & M.EOCENE & 6 & 0 & 0 & 94 & 41 & 10 & 43 & 32 & 11 & 57 & & & & & & & $\mathrm{x}$ & \\
\hline $53-4 / 135-140$ & 490 & E.EOCENE & 11 & 7 & 0 & 82 & 31 & 2 & 49 & 38 & 3 & 58 & & & & & & $\mathrm{x}$ & $\mathrm{x}$ & \\
\hline
\end{tabular}

a Abbreviations as in Table 1. 
Table 3. Bulk and clay mineralogy, Leg 110, Hole 674A.

\begin{tabular}{|c|c|c|c|c|c|c|c|c|c|c|c|c|c|c|c|c|c|c|c|c|}
\hline \multirow[t]{2}{*}{ SAMPLE } & \multirow{2}{*}{$\begin{array}{l}\text { DEPTH } \\
\text { (mbsf) }\end{array}$} & \multirow[t]{2}{*}{ AGE } & \multicolumn{4}{|c|}{ BULK MINERALOGY } & \multicolumn{3}{|c|}{ BULK CLAY MINERALOGY } & \multicolumn{3}{|c|}{ CLAY-SIZED FRACTION } & \multicolumn{8}{|c|}{ ACCESSORY MINERALS } \\
\hline & & & QTZ & PLAG & $\mathrm{CAL}$ & CLAY & SM & IL & $\mathrm{K}+\mathrm{C}$ & SM & IL & $\mathrm{K}+\mathrm{C}$ & PALY & CLIN & GIBB & $\mathrm{OP}-\mathrm{CT}$ & $\mathrm{DOL}$ & RHOD & AMPH & PYX \\
\hline $1-3 / 140-145$ & 4 & E.PLEIS & 15 & 8 & 7 & 70 & 43 & 11 & 16 & 61 & 16 & 23 & & & & & & & $\mathrm{x}$ & \\
\hline $2-6 / 140-145$ & 14 & E.PLEIS & 8 & 13 & 0 & 78 & 50 & 8 & 20 & 74 & 5 & 21 & & $\mathrm{x}$ & & & & & $\mathrm{x}$ & \\
\hline $3-5 / 140-145$ & 22 & E.PLEIS & 11 & 30 & 0 & 59 & 45 & 2 & 12 & 70 & 5 & 24 & & & & & & & $\mathrm{x}$ & \\
\hline $4-3 / 140-145$ & 30 & E.PLEIS & 9 & 22 & 0 & 68 & 52 & 6 & 11 & 83 & 4 & 13 & & $\mathrm{x}$ & & & & & $\mathrm{x}$ & \\
\hline $5-4 / 140-145$ & 41 & E.PLEIS & 11 & 5 & 42 & 41 & 15 & 10 & 16 & 54 & 16 & 30 & & & & & & $\mathrm{xX}$ & $\mathrm{x}$ & \\
\hline $7-2 / 140-144$ & 56 & L.PLIOC & 14 & 21 & 30 & 36 & 14 & 10 & 12 & 55 & 13 & 32 & & & $\mathrm{x}$ & & & $\mathrm{x}$ & $\mathrm{x}$ & \\
\hline $9-6 / 139-144$ & 81 & $?$ & 8 & 15 & 0 & 76 & 51 & 12 & 14 & 73 & 10 & 17 & & & & & & & $\mathrm{x}$ & \\
\hline $12-3 / 135-140$ & 105 & L.PLIOC & 13 & 19 & 39 & 29 & 16 & 5 & 8 & 67 & 13 & 20 & & & & & & $\mathrm{x}$ & $\mathrm{x}$ & \\
\hline $15-2 / 135-140$ & 132 & $?$ & 8 & 20 & 0 & 72 & 45 & 10 & 16 & 67 & 18 & 16 & & & & & & $\mathrm{x}$ & $\mathrm{x}$ & \\
\hline $18-5 / 133-139$ & 165 & $?$ & 12 & 17 & 0 & 71 & 42 & 10 & 19 & 77 & 8 & 16 & & $\mathrm{x}$ & & & & $\mathrm{x}$ & $\mathrm{x}$ & \\
\hline $21-3 / 135-140$ & 191 & $?$ & 8 & 10 & 0 & 83 & 57 & 9 & 16 & 67 & 12 & 21 & & $\mathrm{x}$ & & & & $\mathrm{x}$ & $\mathrm{x}$ & \\
\hline $24-5 / 135-140$ & 222 & $?$ & 10 & 21 & 0 & 69 & 58 & 4 & 7 & 87 & 5 & 8 & & & & & & & $\mathrm{x}$ & \\
\hline $27-5 / 135-139$ & 251 & $?$ & 13 & 5 & 0 & 82 & 43 & 9 & 29 & 61 & 10 & 29 & & & & & & $\mathrm{x}$ & $\mathrm{x}$ & \\
\hline $30-3 / 135-140$ & 276 & M.EOCENE & 19 & 0 & 21 & 60 & 45 & 2 & 13 & 77 & 2 & 21 & & $\mathrm{x}$ & & & & & $\mathrm{x}$ & \\
\hline $33-5 / 134-139$ & 308 & E.OLIGO & 4 & 5 & 0 & 91 & 81 & 3 & 7 & 91 & 3 & 6 & & & & & & $\mathrm{x}$ & $\mathbf{x}$ & \\
\hline $36-5 / 135-139$ & 333 & $?$ & 6 & 4 & 0 & 90 & 72 & 5 & 13 & 63 & 8 & 29 & & & & & & $\mathrm{x}$ & $\mathrm{x}$ & \\
\hline $39-5 / 136-140$ & 365 & E.OLIGO & 6 & 2 & 0 & 92 & 61 & 1 & 30 & 59 & 4 & 37 & & & & & & & $\mathrm{x}$ & $\mathrm{x}$ \\
\hline $41-4 / 115-120$ & 382 & E.OLIGO & 8 & 3 & 0 & 89 & 59 & 3 & 27 & 66 & 4 & 30 & & & & & & $\mathrm{x}$ & $\mathrm{x}$ & \\
\hline $42-3 / 135-140$ & 390 & $?$ & 10 & 2 & 0 & 88 & 50 & 6 & 32 & 62 & 4 & 34 & & $\mathrm{x}$ & & & & $\mathrm{x}$ & $\mathrm{x}$ & \\
\hline $45-4 / 133-137$ & 420 & $?$ & 5 & 3 & 0 & 93 & 77 & 4 & 12 & 71 & 4 & 25 & & $\mathrm{x}$ & & & & $\mathrm{x}$ & $\mathrm{x}$ & \\
\hline $48-3 / 127-130$ & 447 & $?$ & 13 & 8 & 0 & 78 & 70 & 2 & 7 & 89 & 2 & 9 & & & & & & & $\mathrm{xX}$ & \\
\hline
\end{tabular}

a Abbreviations as in Table 1. 


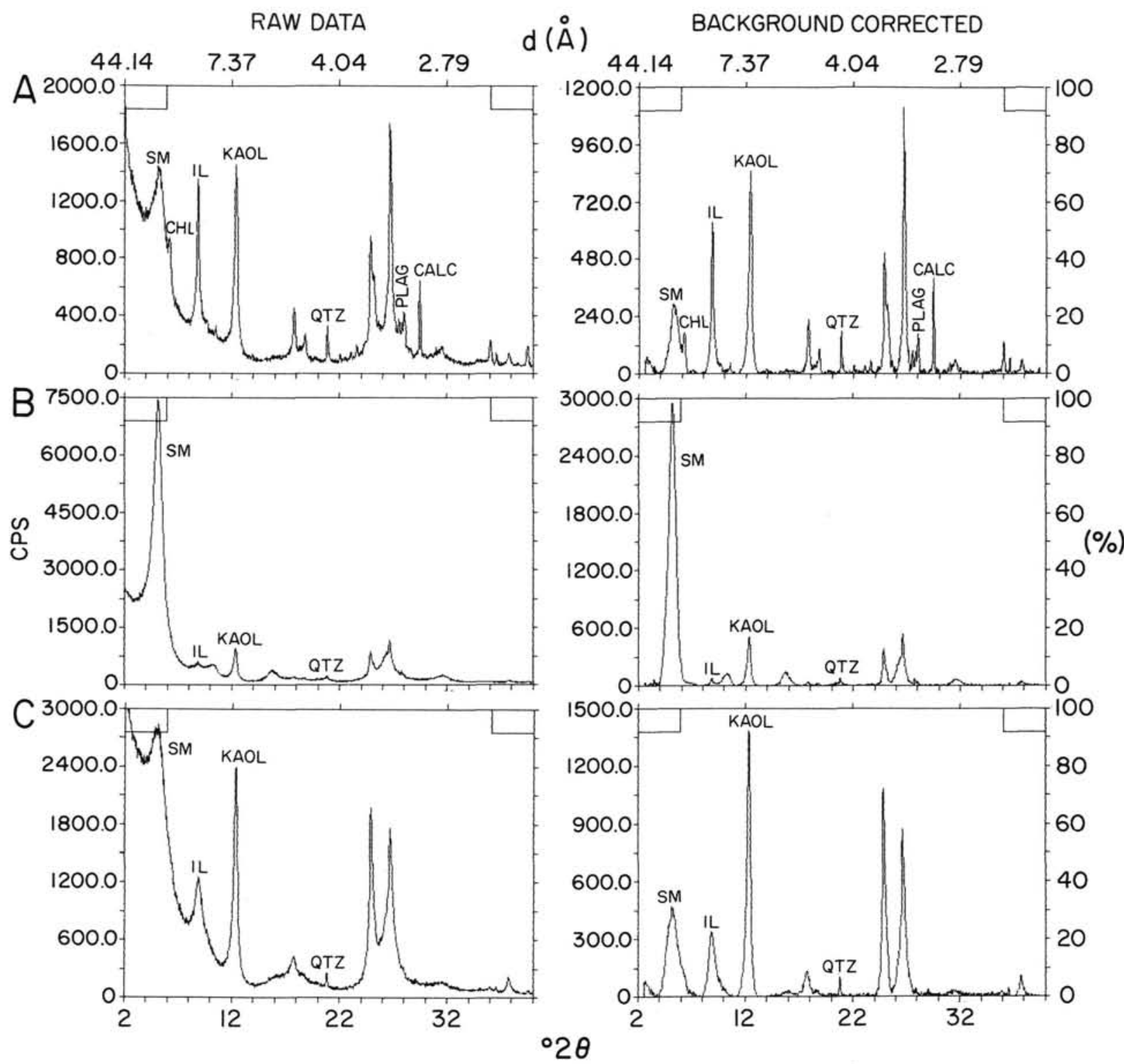

Figure 2. Representative diffraction profiles from Hole 671B. Both raw data and background corrected patterns are shown. Most of the major peaks for smectite (SM), illite (IL), kaolinite (KAOL), chlorite (CHL), quartz (QTZ), plagioclase (PLAG), and calcite (CALC) are labeled. A. Sample from Core 110-671B-18-4 (163 mbsf) is typical of the Pliocene-Pleistocene sediments from Hole 671B. B. The smectite-rich Miocene section is represented by this sample from Core 110-671B-46-3 (420 mbsf). The smectite has a very low percentage of illite interlayers. C. This lower Oligocene sediment (Core 110-671B-67-5, 622 mbsf) contains smectite with about $60 \%$ illite interlayers.

within these intervals (Table 1). A secondary factor causing a decrease in the percentage of total clay is the relatively high plagioclase content of two of the three samples from the proto-décollement zone.

A second contrast with Hole 671B is apparent from comparison of the percent smectite and porosity trends of Hole $672 \mathrm{~A}$. The section from approximately $125-200 \mathrm{mbsf}$ is characterized by generally elevated porosities. Furthermore, the zone of the proto-décollement contains a local porosity maximum. Although smectite content may be responsible, at least in part, for the overall trend of elevated porosities, it cannot explain the local porosity maximum in the proto-décollement zone. The sediments of this zone contain minor amounts of radiolaria that may account for some excess porosity (see the Site Summary chapters, Mascle, Moore, et al., 1988). The overlying smectiterich sediments of Hole $672 \mathrm{~A}$, as well as the sediments above and within the décollement at Hole $671 \mathrm{~B}$, are free of radiolarians. Radiolarian content at Hole 672A, however, steadily increases downward from the proto-décollement to about $208 \mathrm{mbsf}$, whereas porosity decreases over this depth range. Other factors must be playing a role in porosity determination in these sediments.

Analogous plots of percent smectite, total clay content, porosity, and fault location for Hole $674 \mathrm{~A}$ are not very informative because of the few samples analyzed and the structual complexity of that site, and are therefore not included here.

\section{Extent of Mixed Layering in the Smectite}

As mentioned above, smectite minerals in Leg 110 sediments may be derived from either volcanic ash from the Lesser Antilles Arc or continental weathering. The nature of the occurrence and the composition of the smectite in terms of percent of 

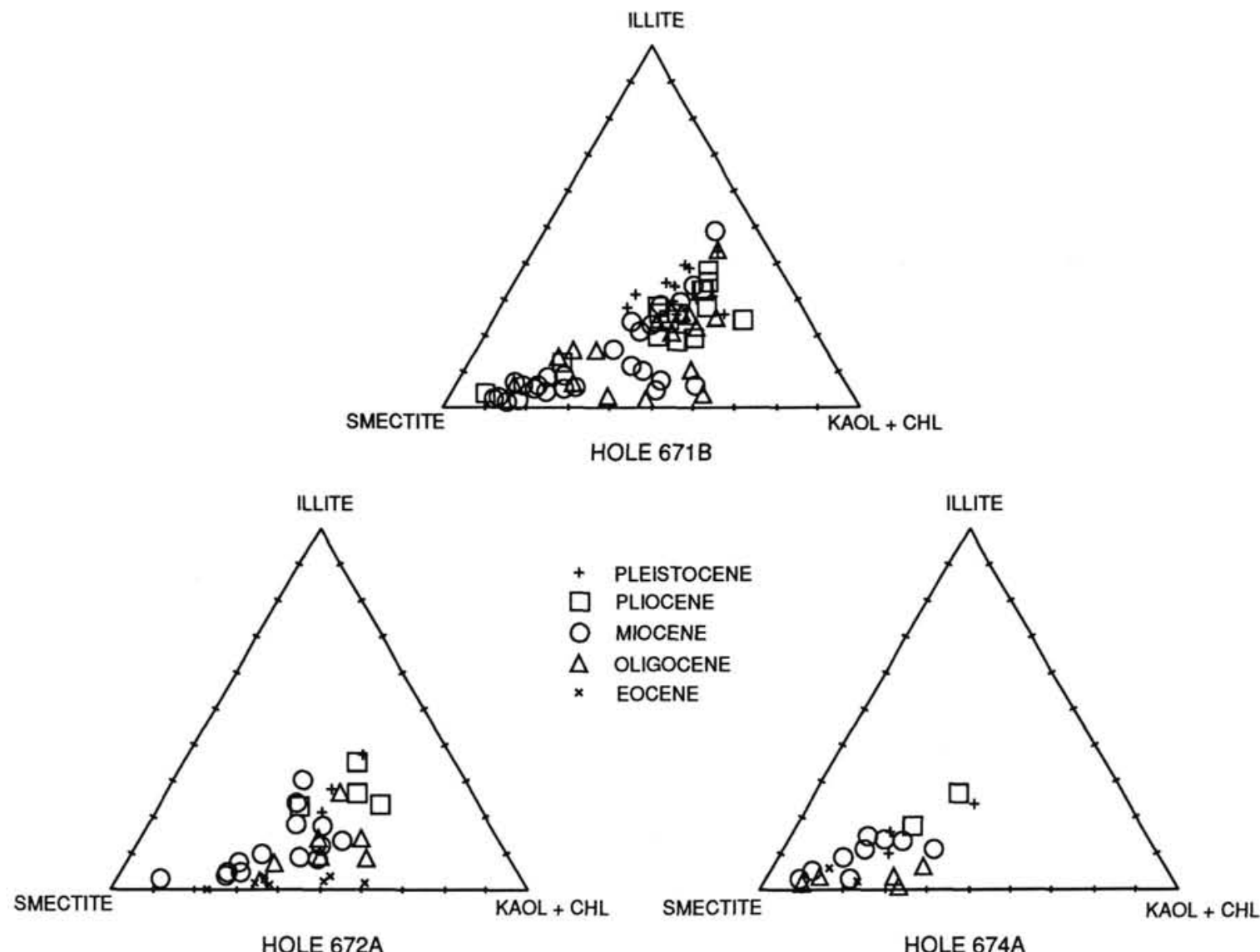

Figure 3. Variations in clay mineralogy as a function of age in sediments from Holes 671B, 672A, and 674A plotted on triangular diagrams. The percentages of smectite, illite, and kaolinite plus chlorite for the bulk samples in Tables 1, 2, and 3 have been normalized to 100 .

mixed layering can provide an indication of source. Ash layers are characterized by low total clay content relative to surrounding sediments, but most of the clay is smectite. As the ash layers are altered, the amount of clay increases such that both percent total clay and percent smectite are greater in the ash layer than in the surrounding sediment (Hein and Scholl, 1978). Vallier and Kidd (1977) suggested that $>90 \%$ smectite in the clay fraction of a sediment indicates that sediment is of volcanic origin. A sediment containing $75-90 \%$ smectite is mostly of volcanic origin, and one containing 50-75\% smectite probably has some volcanic input. Zeolites are also common alteration products of volcanic ash (Hay, 1966; Stonecipher, 1976). Although not diagnostic, the coexistence of zeolites with smectite is consistent with a volcanogenic origin of the sediment.

The relatively high total clay content and percent smectite of the Miocene sections in Holes 671B and 672A and the Eocene sediments from Hole $672 \mathrm{~A}$ suggest that these sediments contain a significant primary volcanogenic component (Figs. 4 and 5). The presence of the zeolite clinoptilolite supports this assertion (Tables 1 and 2). Figure 6 shows the percent smectite in the clay fraction of the sediments vs. depth for Holes 671B, 672A, and $674 \mathrm{~A}$. The shapes of the plots are very similar to those of percent smectite in the bulk sediment (Figs. 4 and 5). Percent smectite is low throughout the Pliocene-Pleistocene section of Hole 671B (from about 0 to $300 \mathrm{mbsf}$ ), but increases slightly with depth. This trend probably reflects progressive alteration of volcanic ash to smectite with depth. The Miocene section in Hole $671 \mathrm{~B}$ generally contains greater than $50 \%$, and frequently greater than $75 \%$, smectite in the clay fraction, indicating significant volcanogenic influence. The Oligocene sediments have lower, but quite variable, smectite contents. In Hole $672 \mathrm{~A}$, the trends of percent smectite in the clay fraction are similar to those at Hole 671B, with Miocene and Eocene sediments showing the greatest contribution of volcanogenic debris. The samples analyzed from Hole $674 \mathrm{~A}$ all contain more than $50 \%$, and many more than $75 \%$, smectite in the clay-size fraction. This indication of significant volcanogenic input to all the sediments of Site 674 is in agreement with the slightly higher plagioclase contents of these sediments relative to those of Sites 671 and 672, as noted above.

The composition of the smectite, in terms of degree of mixed layering, is also indicative of source. Smectite formed from degradation of volcanic ash is generally a dioctahedral montmorillonite that has few illite interlayers. In contrast, smectites formed during continental weathering commonly have $40-50 \%$ illite interlayers. Perry et al. (1976a) distinguished volcanically-derived from continentally-derived smectite in Caribbean sediments from DSDP Site 149 on the basis that smectite derived from submarine alteration of ash has less than $10 \%$ interlayered illite whereas that delivered from the continent contains $40 \%$ illite interlayers on the average. Similar results have been obtained for sediments from the Bering Sea (Hein et al., 1976) and the Bellinghausen Abyssal Plain and continental rise off the Antarctic Peninsula (Perry et al., 1976b).

The degree of mixed layering of smectite may reflect diagenesis rather than source, however. Increases in the percentage of illite interlayers in mixed-layer illite/smectite during burial have been well documented from a number of environments (e.g., 


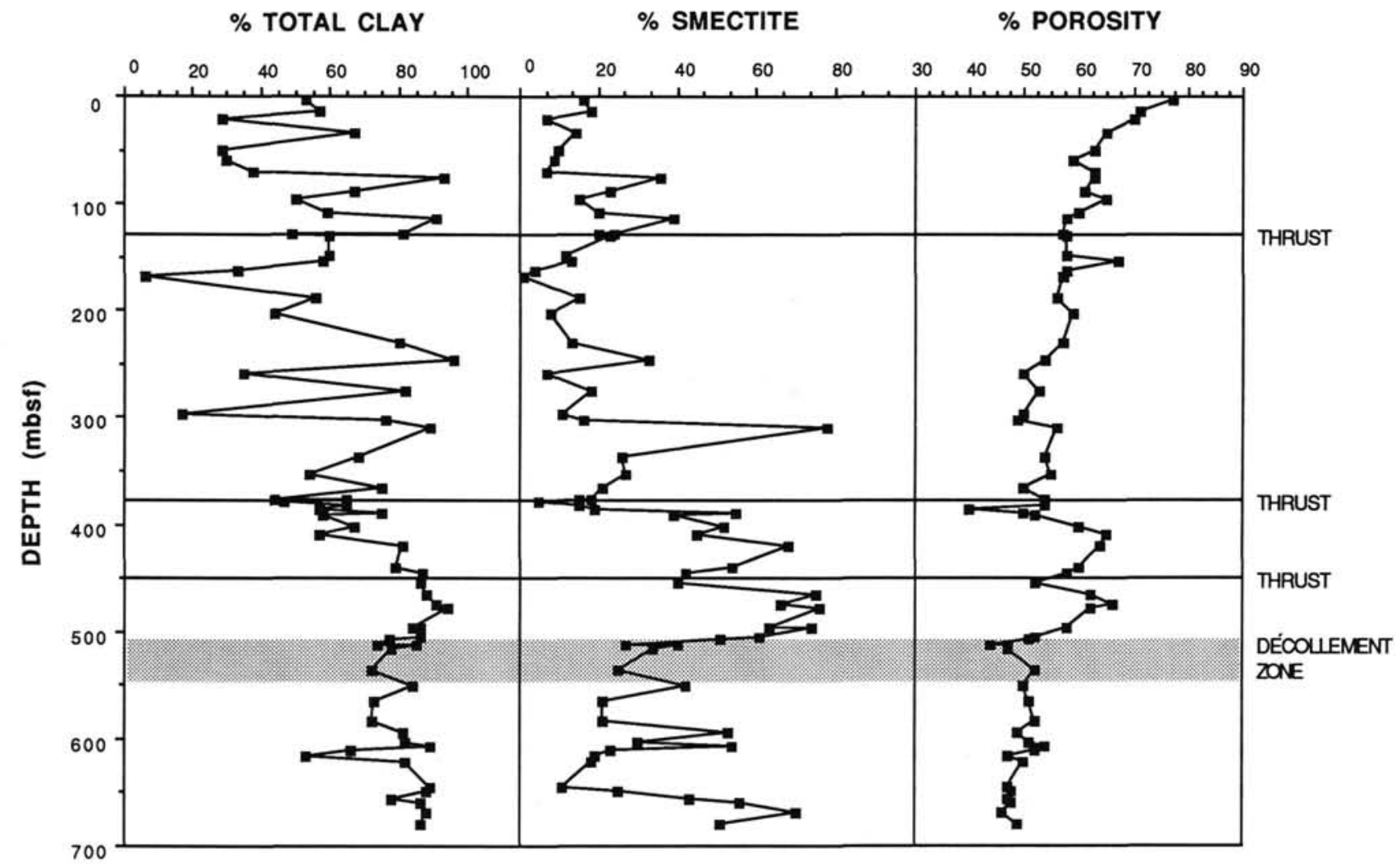

Figure 4. Comparison of the depth trends of percent total clay, percent smectite, and porosity for Hole 671B. Locations of the major thrust faults and of the décollement zone are marked. The thrusts, and particularly the décollement, are located in sections underlying local highs in percent smectite. The décollement also formed in sediments of relatively low porosity, immediately beneath a section of elevated porosity.

Dunoyer de Segonzac, 1970; Hower et al., 1976; Hoffman and Hower, 1979; Schoonmaker et al., 1986). A general reaction for conversion of smectite interlayers to illite can be written

$$
\text { smectite }+\mathrm{K}^{+} \rightarrow \text { illite + cations + silica + water }
$$

(Hower et al., 1976; Boles and Franks, 1979). These studies have concluded that conversion of smectite layers in illite/smectite to illite is dependent primarily on temperature, with fluid and solid phase compositions, pressure, and time playing lesser, but significant roles. The reaction progresses from the composition of the detrital illite/smectite through a series of intermediate compositions to the eventual pure illite product. Although reaction rates vary significantly from site to site, temperatures in the range $60-90^{\circ} \mathrm{C}$ are generally required for conversion of detrital illite/smectite to a composition of about $40 \%$ smectite interlayers (e.g., Dunoyer de Segonzac, 1970; Hower et al., 1976; Boles and Franks, 1979; Chang et al., 1986).

The degree of mixed layering in the smectite, represented as the percentage of smectite interlayers, is illustrated as a function of depth for Holes 671B, 672A, and 674A in Figure 7. Most samples contain relatively pure smectite having fewer than $30 \%$ illite interlayers. These smectites are most likely formed authigenically by submarine alteration of volcanic ash, although some samples may include a little detrital smectite derived from South America. The pure smectite found by Schoonmaker (1986) in Leg 78A sediments is also most likely a fresh alteration product of volcanic ash. That smectite was originally identified as saponite, a trioctahedral smectite, but is now thought to be a dioctahedral smectite with some trioctahedral character. The pure smectite was found preferentially in sediments above the décollement and along a thrust fault, in agreement with the findings here of high smectite contents associated with these zones. The fact that pure smectite was not found in samples from the reference Site 543 may be an artifact owing to the relatively small number of samples analyzed. The most notable exception to the relative uniformity of smectite composition is in Hole 671B. There is a sharp decrease in the percentage of smectite interlayers (an increase in the degree of mixed layering) at about $500 \mathrm{mbsf}$, the depth of the décollement. Within the décollement zone and the underthrust sequence, the composition of the smectite varies irregularly between 32 and $80 \%$ smectite interlayers, with most samples containing fewer than $65 \% \mathrm{smec}-$ tite interlayers. If this compositional variation is a result of influx of detrital illite/smectite from South America, the stratigraphically equivalent section from Hole 672A should show similar compositions. The sediments of the proto-décollement and the underlying upper Oligocene sediments from Hole 672A, however, contain smectite with few illite interlayers. The smectite in every sample analyzed from Holes $672 \mathrm{~A}$ and $674 \mathrm{~A}$ appears to be dominantly authigenic in origin.

The above evidence supports the interpretation that the smectites of the décollement and underthrust zones at Hole $671 \mathrm{~B}$ have been diagenetically altered. The driving force for this diagenetic reaction, however, is not clear. Heat flux measurements at this site (Mascle, Moore, et al., 1988; Fisher and Hounslow, this volume) indicate a thermal gradient much too low to account for temperatures required for this reaction in a normal burial diagenetic setting (generally $60-90^{\circ} \mathrm{C}$ ). Although the thermal gradient measured for the shallow portion of the hole was steep $\left(103^{\circ} \mathrm{C} / \mathrm{km}\right.$ at $\left.36 \mathrm{mbsf}\right)$, the gradient determined for the rest of the hole was $43^{\circ} \mathrm{C} / \mathrm{km}$. At a depth of $500 \mathrm{mbsf}$, this gradient yields a temperature of only about $24^{\circ} \mathrm{C}$. Calculations from results of experiments on the kinetics of the illite/ smectite transformation by Eberl and Hower (1976) indicate 


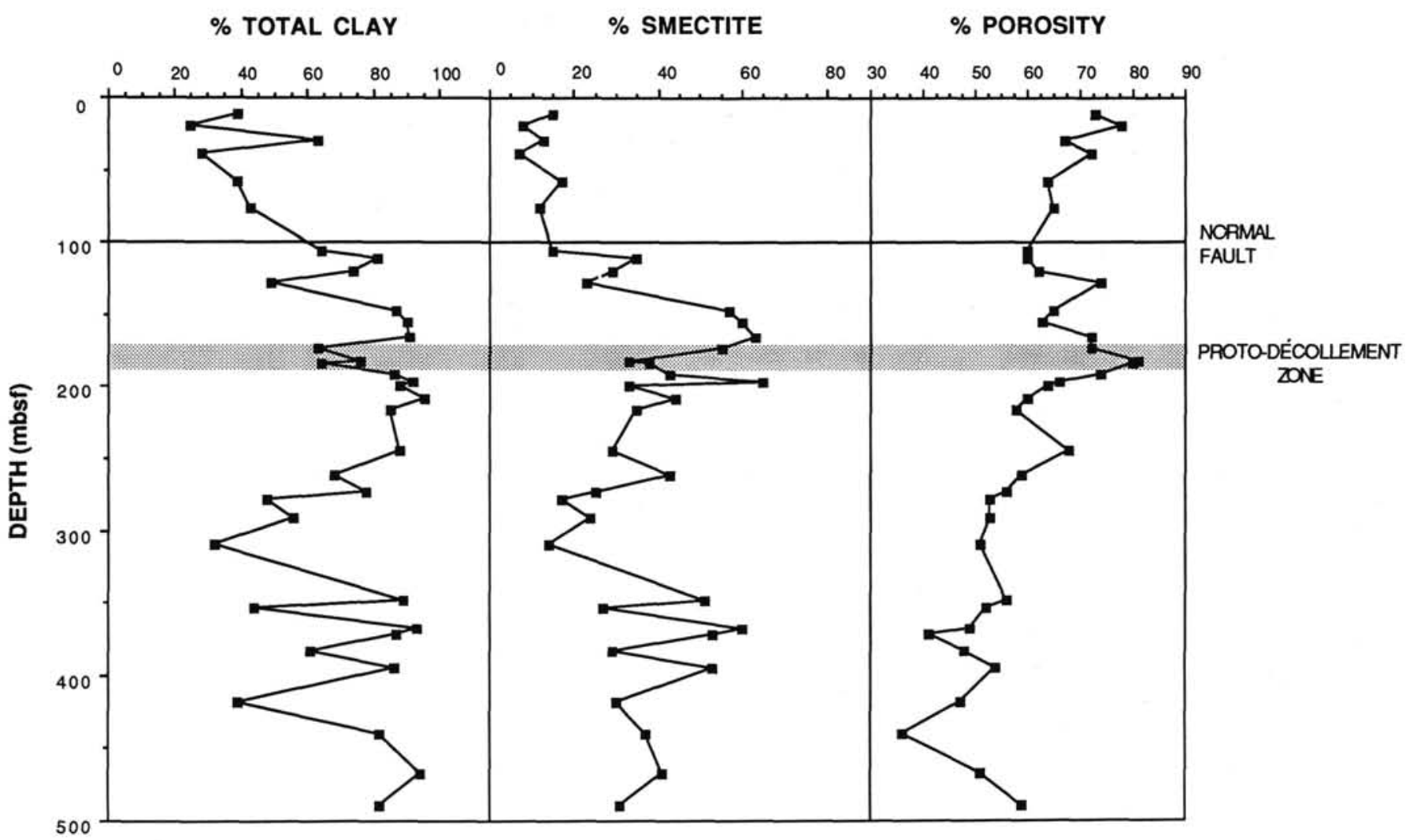

Figure 5. Comparison of the depth trends of total clay, smectite, and porosity for Hole 672A. The proto-décollement zone, indicated by the stipled region, is characterized by low percent total clay and smectite relative to the overlying sediments. In contrast to the décollement zone at Hole 671B, the proto-décollement is a region of locally elevated porosity.

that formation of an illite/smectite with $30 \%$ smectite interlayers at $25^{\circ} \mathrm{C}$ would require on the order of $6 \mathrm{~m}$.y. It appears that fluids from greater depths are being advected through the décollement zone and the underthrust sequence (ODP Leg 110 Scientific Party, 1987; Mascle, Moore, et al., 1988). Migration of fluids from depth may be episodic, but could periodically expose sediments along the migration paths to elevated temperatures. The kinetics of the illite/smectite reaction, however, would require that elevated temperatures be maintained for long time periods: about $6 \mathrm{k} . \mathrm{y}$. at $120^{\circ} \mathrm{C}, 60 \mathrm{k}$.y. at $90^{\circ} \mathrm{C}$, and 700 k.y. at $60^{\circ} \mathrm{C}$.

Although temperature and time are classically considered to be major controls on illitization reactions, other factors also may be important. High permeabilities and water/rock ratios may promote the illite/smectite conversion by increasing the delivery of reactants to the reaction site (Heling, 1974; Ahn et al., 1988). Ahn et al. (1988) suggested that high water/rock ratios, rather than temperature, may be the determining factor in diagenesis of smectite and other phyllosilicates in volcanogenic sediments from New Zealand.

Reaction of the smectite with pore fluids of altered composition offers another possible explanation. Exposure of iron-bearing smectites to reducing solutions can cause reduction of structural iron, thereby increasing the net negative layer charge of the clay (Perry and Hower, 1970; Eslinger et al., 1979). The increased layer charge could favor the fixation of additional $\mathrm{K}^{+}$ and conversion of smectite interlayers to illite. Evidence of the reducing nature of the pore fluids in the décollement zone was provided by the high dissolved Mn concentrations noted in Hole 671C (Mascle, Moore, et al., 1988). Although the concentration of $\mathrm{Fe}^{3+}$ in the smectites has not been determined, the relative intensities of $\mathrm{X}$-ray diffraction peaks for the smectites are indica- tive of the presence of tetrahedral iron $\left(\mathrm{Fe}^{3+}\right.$; R. C. Reynolds, pers. commun.). Eslinger et al. (1979) suggested that this reaction takes place in conjunction with oxidation of organic matter at temperatures of about $60^{\circ} \mathrm{C}$ in the Overthrust Belt of Montana. Iron reduction in association with organic matter oxidation is a common reaction during early diagenesis of marine sediments, however, and is not limited to environments of elevated temperature (e.g., Berner, 1980).

It is interesting that a similar phenomenon was noted in a study of diagenesis of sediments from a well on Barbados Island (Schoonmaker, et al., 1986). The degree of diagenesis of mixed-layer illite/smectite, as indicated by percent smectite interlayers, appeared to be accelerated at a depth corresponding to a major thrust fault. Vitrinite reflection analyses indicated a gradual increase in diagenetic temperature with depth, with a slight offset at the depth of the fault. There was no indication of significantly elevated temperatures along the fault. It was suggested that unusual fluid compositions and/or elevated water/rock ratios within the fault zone might be responsible for the advanced degree of clay diagenesis.

\section{Potential Influence of Clay Diagenesis on Accretionary Complex Hydrology and Dynamics}

Although no major mineralogical changes were noted in the sediments of the décollement zone, the underthrust sequence, or along thrust faults within the accreted sediments, several diagenetic reactions are apparent from the bulk and clay mineralogy. Diagenetic alteration of volcanic ash to smectite (plus clinoptilolite in many samples) is occurring throughout the sediment column. The implications of this diagenetic reaction are clear. The accumulation of volcanic ash, and its subsequent diagenesis, created the smectite-rich horizons associated with zones 
Hole 671B

Hole 672A

Hole 674A
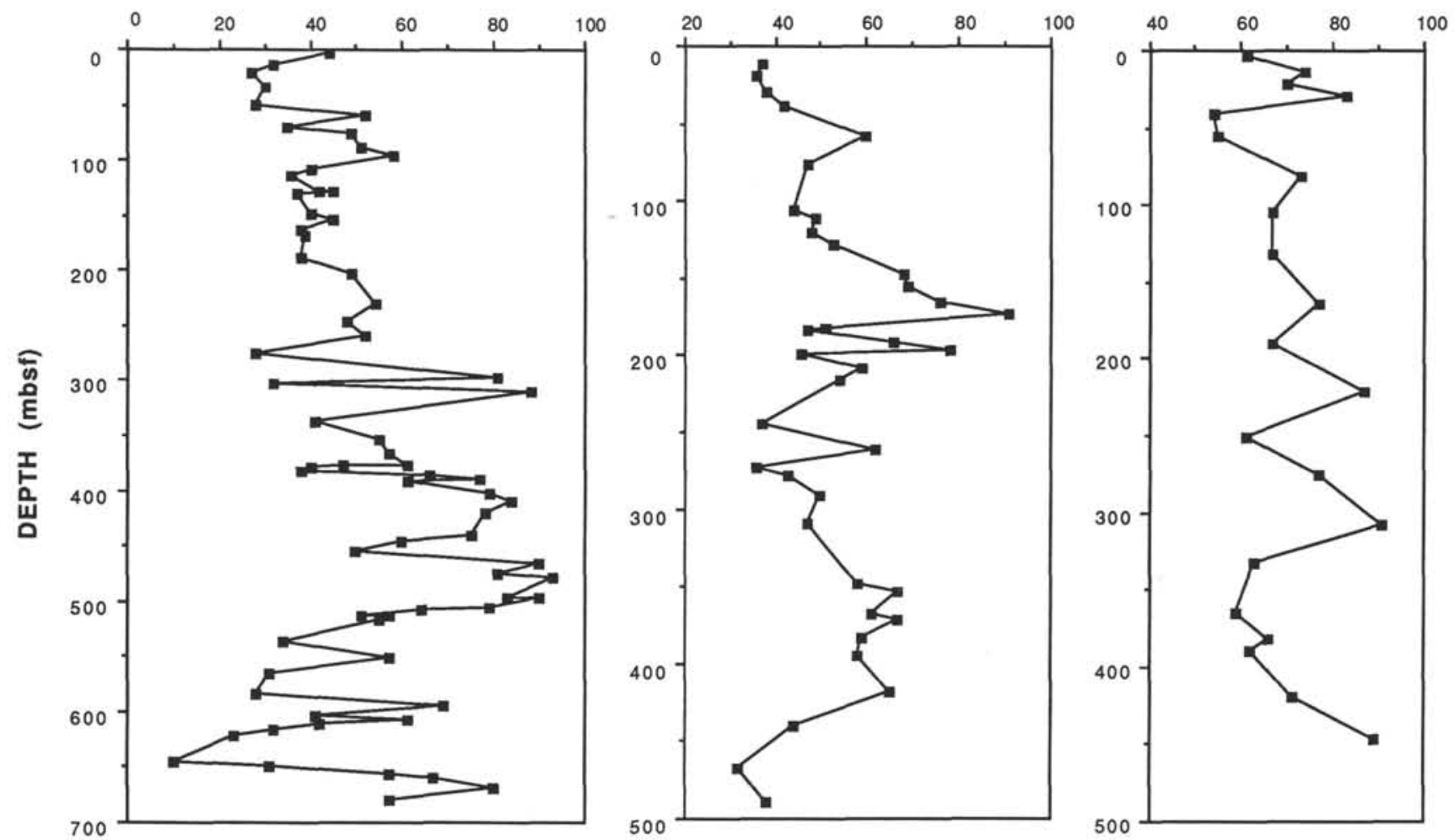

Figure 6. The percentage of smectite in the clay-sized fraction for sediments from Holes 671B, 672A, and 674A. The Miocene sediments from Holes $671 \mathrm{~B}$ and $672 \mathrm{~A}$ and the Eocene section at Hole $672 \mathrm{~A}$ are particularly smectite-rich, indicating the volcanogenic origin of the sediments. All of the samples analyzed from Hole $674 \mathrm{~A}$ appear to have had significant volcanogenic input.

of thrusting and the décollement. The décollement formed directly subjacent to sediments that are largely of volcanic origin and are old enough to have altered to smectite.

The sediments of the décollement zone and the underthrust sequence appear to be undergoing an additional diagenetic reaction, that of conversion of smectite to a mixed-layer illite/smectite. This reaction has important implications with regard to the hydrology and dynamics of the accretionary complex because it involves dehydration of the smectite. The loss of interlayer water from the clay should result in changes in sediment properties (e.g., porosity reduction, increased bulk density, grain density, rigidity, and shear strength) as well as altered pore-water compositions. If the dehydration reactions occur in a sealed zone of low permeability, overpressuring may result (Powers, 1967). It is likely that elevated pore pressures along décollement horizons decrease the shear strength of sediments and facilitate subduction (von Huene and Lee, 1983).

The effect on pore-water compositions of smectite dewatering associated with diagenetic conversion to illite can be estimated. Start with an initial sediment of $35 \%$ porosity whose solid phase consists of $40 \%$ illite/smectite with $80 \%$ smectite interlayers. The initial pore water has the composition of seawater. Conversion of all of the smectite in that sediment to illite releases approximately $23 \mathrm{~g} \mathrm{H}_{2} \mathrm{O} / 1000 \mathrm{~cm}^{3}$ sediment. This release of water accounts for a $4 \%$ freshening of the pore water, assuming constant porosity throughout the diagenetic process. If porosity is reduced during the reaction, a greater freshening will occur. The $\mathrm{Cl}$ anomaly in the décollement zone of Hole 671B indicates freshening of the pore water by approximately $10 \%$. The anomaly at about 367 mbsf in Hole $672 \mathrm{~A}$ is of the same magnitude (Mascle, Moore, et al., 1988). The actual degree of freshening during smectite diagenesis will depend on sediment porosity, and smectite content and composition. It seems reasonable, however, based on the above estimates, that the observed pore-water freshening could be largely the result of smectite dewatering at greater depth in the complex.

In addition to the release of water, the conversion of smectite to illite should be accompanied by uptake of $\mathrm{K}^{+}$and release of various other cations and silica. Interpretation of the pore-water profiles is quite complicated (see Gieskes et al., this volume), but several of the observed depth trends are at least consistent with the production of water as smectite converts to illite. At the depth of the décollement in Hole 671B there is a pronounced maximum in dissolved silica, as well as inflections in the profiles of magnesium (positive) and calcium, sodium, and potassium (negative). Silica also reaches a maximum concentration in the proto-décollement of Hole $672 \mathrm{~A}$. The $\mathrm{Cl}$ anomaly at about 367 mbsf in Hole $672 \mathrm{~A}$ is accompanied by irregular, but generally high, silica concentrations, a minimum in $\mathrm{Na}+\mathrm{K}$, and slight inflections in the calcium and magnesium profiles.

In addition to illite/smectite diagenesis, another possible explanation of pore-water freshening involves membrane filtration. Highly compacted clays can act as semi-permeable membranes that allow passage of water, but exclude ions. Clays such as smectite, with high net surface charges and high cation exchange capacities, make the best geologic membranes (Fritz, 1986). Experimental work on the efficiency of clay minerals as geologic membranes has shown that filtration efficiency varies with cation size and charge, compaction pressure, flow rate, and temperature (e.g., McKelvey and Milne, 1962; Hanshaw and Coplen, 1973; Kharaka and Smalley, 1976). Filtration ratios (ratio of input to output concentration of an ion) for solutions passing through bentonite (altered volcanic ash containing a high proportion of smectite) are generally in the range of 1 to 8 
HOLE 671B

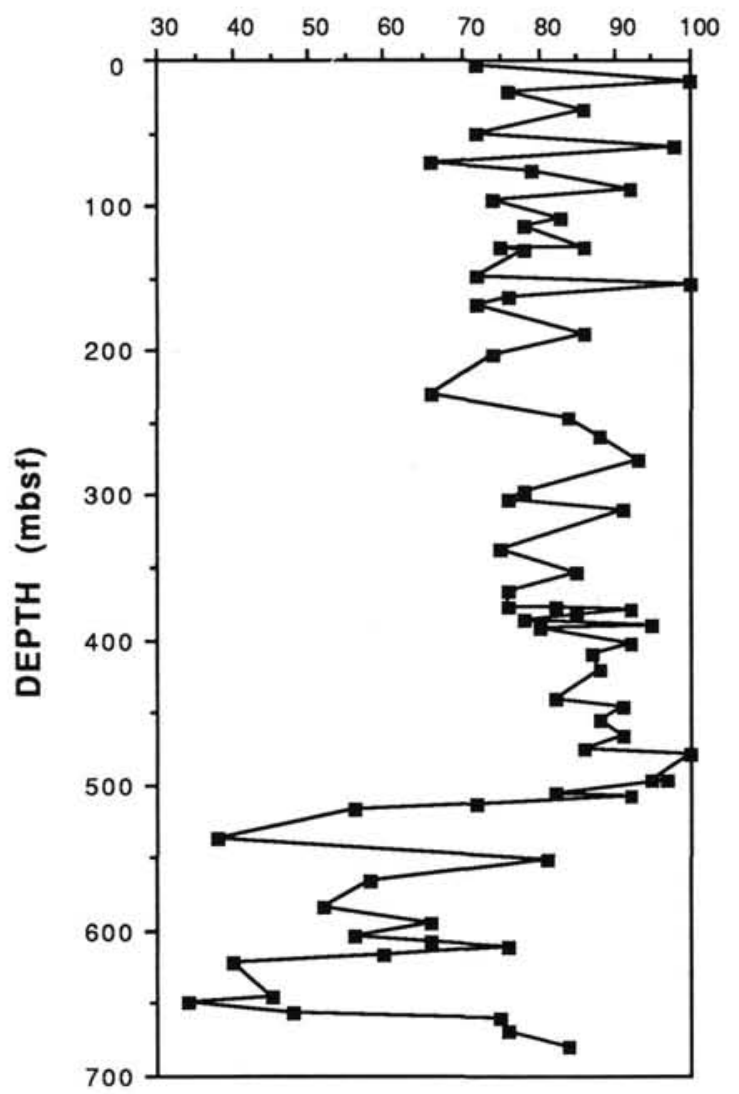

HOLE 672A

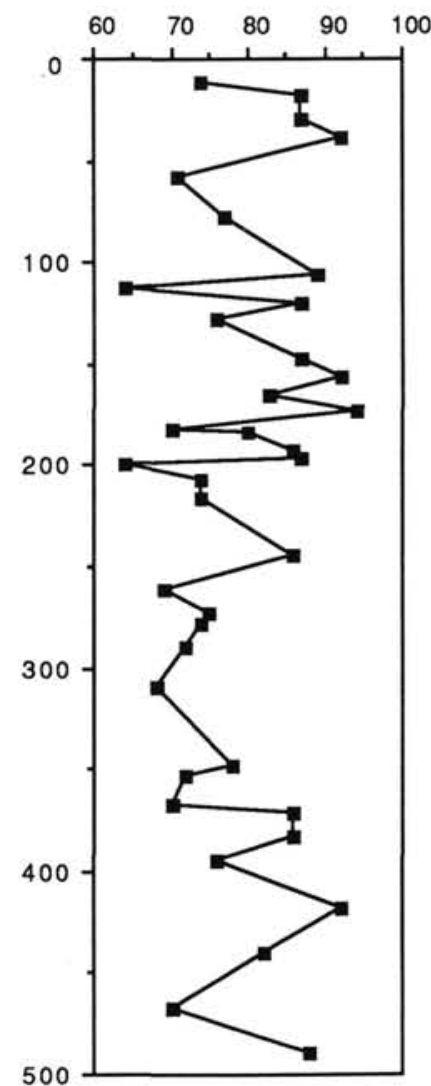

HOLE 674A

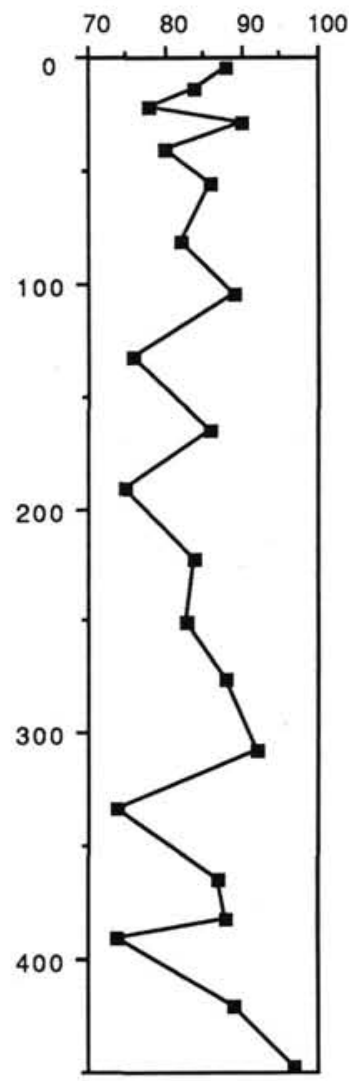

Figure 7. The composition of the smectite, presented as the percentage of smectite interlayers, for sediments from Holes 671B, 672A, and 674A. The high degree of mixed layering (low percentage of smectite interlayers) of the smectite from the décollement and underthrust sequences indicates that these sediments have undergone diagenetic alteration. Note that the stratigraphically equivalent samples from Hole 672 do not show evidence of alteration.

depending on experimental conditions and the ion under consideration (Kharaka and Smalley, 1976). The presence of smectite-rich intervals in the sediments of the accretionary complex and underthrust sequence indicates the likelihood that membrane filtration at depth has altered the composition of Leg 110 pore waters.

Although diagenesis within the sediments sampled during Leg 110 is influencing both fluid and solid phase compositions, it is obvious that the pore-water anomalies observed in the décollement zone and underthrust sequence did not result from in situ diagenetic reactions. The pore fluids clearly are derived from greater depth within the accretionary complex and reflect the integration of diagenetic reactions along their path of migration (Gieskes et al.; Vrolijk et al., both, this volume). Consideration of the clay mineralogy and diagenetic reactions of clay minerals indicates that both clay dehydration associated with illite/smectite diagenesis and membrane filtration influence the composition of pore waters sampled during Leg 110.

\section{SUMMARY}

Clay mineralogy and diagenesis of Leg 110 sediments appear to influence the dynamics of accretion in several ways. Smectite, derived largely from alteration of volcanic ash, contains significant amounts of adsorbed and structural water. Smectite-rich sediments from Leg 110 drill sites tend to have high porosities and low shear strengths, reflecting the relatively high water content of the smectite. The décollement and several major thrust faults at Site 671 formed at boundaries between smectite-rich sediments above and smectite-poor sediments below, presumably because of the contrast in physical properties across the mineralogical boundaries.

Diagenetically altered smectite (illite/smectite) was found in the underthrust sequence of Site 671 , but not in the stratigraphically equivalent sediments of Sites 672 or 674 . These occurrences suggest an in-situ diagenetic, rather than detrital, origin for the illite/smectite. Possible driving forces for the alteration reaction under the relatively low prevailing temperatures include reduction of iron in the smectite by reducing fluids migrating through the décollement and underthrust sequence, and high water/rock ratios in these zones.

Dehydration of smectite occurs during both compaction with burial and diagenetic alteration. At depth within the accretionary complex, dehydration reactions will cause significant changes in sediment physical properties and may influence shifts in the location of the décollement and thrust faults as the complex grows. In addition, water released during smectite dehydration, combined with possible effects of membrane filtration in the clay-rich sediments, will result in freshening of the pore fluids as observed at Leg 110 drilling sites.

\section{ACKNOWLEDGMENTS}

I acknowledge the cooperation and assistance of the crew of the JOIDES Resolution and the ODP technical staff. ODP technician Christian Segade was particularly diligent in shipboard X-ray diffraction, and always with a smile. Christopher Busing and Carol Yonamine provided invaluable laboratory assistance for the shore-based studies. Diane Sakamoto assisted in typing the manuscript and Jozsef Urmos 
helped in preparation of figures. Reviews by W. Johns, F. T. Mackenzie, D. Peacor, M. Underwood, and R. Wilkens helped improve the manuscript. I am grateful for financial support for this study from the U. S. Science Support Program of JOI and the Office of Naval Research.

\section{REFERENCES}

Ahn, J. H., Peacor, D. R., and Coombs, D. S., 1988. Formation mechanisms of illite, chlorite and mixed-layer illite-chlorite in Triassic volcanogenic sediments from the Southland Syncline, New Zealand. Contrib. Mineral. Petrol., 99:82-89.

Berner, R. A., 1980. Early Diagenesis, A Theoretical Approach. Princeton, N.J. (Princeton University Press).

Boles, J. R., and Franks, S. G., 1979. Clay diagenesis in Wilcox sandstones of southwest Texas: implications of smectite diagenesis on sandstone cementation. J. Sediment. Petrol., 49:55-70.

Chang, H. K., Mackenzie, F. T., and Schoonmaker, J., 1986. Comparison between dioctahedral and trioctahedral smectite diagenesis, Brazilian offshore basins. Clays Clay Miner., 34:407-423.

Dunoyer de Segonzac, G., 1970. The transformation of clay minerals during diagenesis and low grade metamorphism: a review. Sedimentology, 15:281-346.

Eberl, D. and Hower, J., 1976. Kinetics of illite formation. Geol. Soc. Am. Bull., 87:1326-1330.

Eslinger, E., Highsmith, P., Albers, D., and deMayo, B., 1979. Role of iron reduction in the conversion of smectite to illite in bentonites in the Disturbed Belt, Montana. Clays Clay Miner., 27:327-338.

Fritz, S. J., 1986. Ideality of clay membranes in osmotic processes: a review. Clays Clay Miner., 34:214-223.

Hanshaw, B. B., and Coplen, T. B., 1973. Ultra-filtration by a compacted clay membrane; II. Sodium ion exclusion at various ionic strengths. Geochim. Cosmochim. Acta, 37:2311-2327.

Hay, R. L., 1966. Zeolites and zeolitic reactions in sedimentary rocks. Geol. Soc. Am. Spec. Pap., 85.

Hein, J. R., and Scholl, D. W., 1978. Diagenesis and distribution of late Cenozoic volcanic sediment in the southern Bering Sea. Geol. Soc. Am. Bull., 89:197-210.

Hein, J. R., Scholl, D. W., and Gutmacher, C. E., 1976. Neogene clay minerals of the far NW Pacific and southern Bering Sea. In Bailey, S. W. (Ed.), A.I.P.E.A. Proceedings, 1975 International Clay Conference, Mexico City: Illinois (Applied Publishing), 71-80.

Heling, D., 1974. Diagenetic alteration of smectite in argillaceous sediments of Rhine graben (SW Germany). Sedimentology, 21:463-472.

Hoffman, J., and Hower, J., 1979. Clay mineral assemblages as low grade metamorphic geothermometers: application to the thrust faulted disturbed belt of Montana, USA. Soc. Econ. Paleontol. Mineral. Spec. Publ., 26:55-79.

Horton, D. G., 1983. Argillic alteration associated with the Amethyst vein system, Creede Mining District. [Ph.D. dissert.]. University of Illinois, Urbana.

Hower, J., Eslinger, E., Hower, M. E., and Perry, E. A., 1976. Mechanism of burial metamorphism of argillaceous sediments: I-mineralogical and chemical evidences. Geol. Soc. Am. Bull., 87:725-737.

Kharaka, Y. K., and Smalley, W. C., 1976. Flow of water and solutes through compacted clays. AAPG Bull. , 60:973-980.

Mann, U. and Müller, G., 1979. X-ray mineralogy of Deep Sea Drilling Projects Legs 51 through 53, Western North Atlantic. In Donnelly, T., Francheteau, J., et al., Init. Repts. DSDP 51, 52, 53, Pt. 2: Washington (U.S. Govt. Printing Office), 721-729.

Marlow, M. S., Lee, H. J., and Wright, A., 1984. Physical properties of sediment from the Lesser Antilles Margin along the Barbados Ridge: Results from Deep Sea Drilling Project Leg 78A. In Biju-Dural, B., and Moore, J. C., et al., Init. Repts. DSDP, 78A: Washington (U.S. Govt. Printing Office), 549-558.

Mascle, A., Moore, J. C., et al., 1988. Proc. ODP, Init. Repts., 110: College Station, TX (Ocean Drilling Program).

McKelvey, J. G., and Milne, I. H., 1962. The flow of salt through compacted clays. Clays Clay Miner., 9:248-259.
Mehra, O. P., and Jackson, M. L., 1960. Iron oxide removal from soils and clays by a dithionite-citrate system buffered with sodium bicarbonate. In Swineford, Ada (Ed.), Clays and Clay Minerals, Proc. 7th Natl. Conf., Washington, D.C., 1958: New York (Pergamon Press), 317-327.

Moore, J. C., Biju-Duval, B. et al., 1984. Tectonic synthesis, Deep Sea Drilling Project Leg 78A, structural evolution of offscraped and underthrust sediment, northern Barbados ridge complex. In BijuDuval, B., and Moore, J. C., et al., Init. Repts. DSDP, 78A: Washington (U.S. Govt. Printing Office), 601-621.

Nadeau, P. H., and Reynolds, R. C., 1981. Burial and contact metamorphism in the Mancos shale. Clays Clay Miner., 29:249-259.

Natland, J. H., 1984. Occurrences of air-fall volcanic ash derived from the Lesser Antilles Arc at Leg 78A drill sites. In Biju-Duval, B., and Moore, J. C., et al., Init. Repts. DSDP, 78A: Washington (U.S. Govt. Printing Office), 369-375.

ODP Leg 110 Scientific Party, 1987. Expulsion of fluids from depth along a subduction-zone decollement horizon. Nature, 236:785-788.

Perry, E., Beckles, E. C., and Newton, R. M., 1976a. Chemical and mineralogical studies, sites 322 and 325 . In Hollister, C. D., Craddock, C., et al., Init. Repts. DSDP, 35: Washington (U. S. Govt. Printing Office), $465-469$.

Perry, E., Gieskes, J. M., and Lawrence, J. R., 1976b. Mg, Ca, and ${ }^{18} \mathrm{O} /{ }^{16} \mathrm{O}$ exchange in the sediment pore water system, Hole 149 , DSDP. Geochim. Cosmochim. Acta, 40:413-423.

Perry, E., and Hower, J., 1970. Burial diagenesis in Gulf Coast pelitic sediments. Clays and Clay Minerals, 18:165-177.

Powers, M. C., 1967. Fluid-release mechanisms in compacting marine mud rocks and their importance in oil exploration. AAPG Bull., 51 : $1240-1254$.

Pudsey, C. J., 1984. X-ray mineralogy of Miocene and older sediments from Deep Sea Drilling Project Leg 78A. In Biju-Duval, B., Moore, J. C., et al., Init. Repts. DSDP, 78A: Washington (U.S. Govt. Printing Office), 325-342.

Reynolds, R. C., Jr., and Hower, J., 1970. The nature of interlayering in mixed-layer illite-montmorillonites. Clays Clay Miner., 18:25-36.

Schoonmaker, J., 1986. Clay mineralogy and diagenesis of sediments from deformation zones in the Barbados accretionary prism (DSDP Leg 78A). In Moore, J. C. (Ed.), Synthesis of Structural Fabrics of Deep Sea Drilling Project Cores from Forearcs. Mem. Geol. Soc. Am., 166:105-116.

Schoonmaker, J., Mackenzie, F. T., and Speed, R. C., 1986. Tectonic implications of illite/smectite diagenesis, Barbados accretionary prism. Clays Clay Miner., 34:465-472.

Stonecipher, S. A., 1976. Origin, distribution and diagenesis of phillipsite and clinoptilolite in deep-sea sediments. Chem. Geol., 17:307318.

Vallier, T. L., and Kidd, R. B., 1977. Volcanogenic sediments in the Indian Ocean. In Heirtzler, J. R. (Ed.), Indian Ocean Geology and Biostratigraphy: Washington (Am. Geophys. Union), 87-118.

Vergo, N., 1984. Wallrock alteration at the Bulldog Mountain Mine, Creede Mining District, Colorado. [M.S. thesis]. University of Illinois, Urbana.

von Huene, R., and Lee, H., 1983. The possible significance of pore fluid pressures in subduction zones. In Watkins, J. S., and Drake, C. L. (Eds.), Studies in Continental Margin Geology. AAPG Mem., 34:781-791.

Weaver, C. E., and Beck, K. C., 1971. Clay water diagenesis during burial, how mud becomes gneiss. Geol. Soc. Am. Spec. Pap., 134.

Wright, A., 1984. Sediment distribution and depositional processes operating in the Lesser Antilles intraoceanic island arc, Eastern Caribbean. In Biju-Duval, B., and Moore, J. C., et al., Init. Repts. DSDP, 78A: Washington (U.S. Govt. Printing Office), 301-324.

Date of initial receipt: 3 June 1988

Date of acceptance: 20 April 1989

Ms 110B-131 